\title{
The Gaia-ESO Survey: Oxygen Abundance in the Galactic Thin and Thick Disks*
}

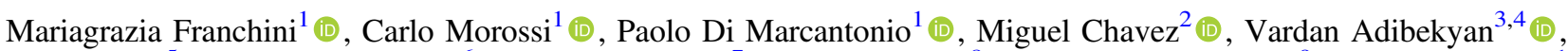 \\ Thomas Bensby $^{5}$ (D), Angela Bragaglia ${ }^{6}$ (D), Anais Gonneau ${ }^{7}$ (D), Ulrike Heiter ${ }^{8}$ (D), Georges Kordopatis ${ }^{9}$ (D), Laura Magrini ${ }^{10}$ (D) $^{\text {, }}$, \\ Donatella Romano $^{6}$ (D) , Luca Sbordone ${ }^{11}$ (D), Rodolfo Smiljanic ${ }^{12}$ (D) , Gražina Tautvaišiene $\dot{e}^{13}$ (D), Gerry Gilmore ${ }^{7}$ (D), \\ Sofia Randich ${ }^{14}$ (D), Amelia Bayo ${ }^{15,16}$ (D), Giovanni Carraro ${ }^{17}$ (D), Lorenzo Morbidelli ${ }^{10}$ (D), and Simone Zaggia ${ }^{18}$ (ID) \\ ${ }_{1}^{1}$ INAF-Osservatorio Astronomico di Trieste, Via G. B. Tiepolo 11, Trieste, I-34143, Italy; mariagrazia.franchini@inaf.it \\ ${ }^{2}$ Instituto Nacional de Astrofísica, Óptica y Electrónica, Luis Enrique Erro 1, 72840 Tonantzintla, Puebla, Mexico \\ ${ }^{3}$ Instituto de Astrofísica e Ciências do Espaço, Universidade do Porto, CAUP, Rua das Estrelas, 4150-762 Porto, Portugal \\ ${ }^{4}$ Departamento de Física e Astronomia, Faculdade de Ciências, Universidade do Porto, Rua do Campo Alegre, 4169-007 Porto, Portugal \\ ${ }^{5}$ Lund Observatory, Department of Astronomy and Theoretical Physics, Box 43, SE-221 00 Lund, Sweden \\ ${ }^{6}$ INAF-Osservatorio di Astrofisica e Scienza dello Spazio di Bologna, Via Gobetti 93/3 I-40129 Bologna, Italy \\ ${ }^{7}$ Institute of Astronomy, University of Cambridge, Madingley Road, Cambridge CB3 0HA, UK \\ ${ }^{8}$ Observational Astrophysics, Department of Physics and Astronomy, Uppsala University, Box 516, SE-75120 Uppsala, Sweden \\ ${ }^{9}$ Université Côte d'Azur, Observatoire de la Côte d'Azur, Laboratoire Lagrange, CNRS UMR 7293 CS 34229 F-06304 Nice Cedex 04, France \\ ${ }^{10}$ INAF-Osservatorio Astrofisico di Arcetri, Largo E. Fermi 5, Florence I-50125, Italy \\ ${ }^{11}$ European Southern Observatory, Alonso de Cordova 3107 Vitacura, Santiago de Chile, Chile \\ ${ }^{12}$ Nicolaus Copernicus Astronomical Center, Polish Academy of Sciences, ul. Bartycka 18, 00-716, Warsaw, Poland \\ ${ }^{13}$ Institute of Theoretical Physics and Astronomy, Vilnius University, Sauletekio av. 3, 10258, Vilnius, Lithuania \\ ${ }^{14}$ INAF-Osservatorio Astrofisico di Arcetri, Largo E. Fermi 5, I-50125, Florence, Italy \\ ${ }^{15}$ Instituto de Física y Astronomía, Universidad de Valparaíso, Avda. Gran Bretaña 1111, Valparaíso, Chile \\ ${ }^{16}$ Núcleo Milenio de Formación Planetaria, NPF, Universidad de Valparaíso, Chile \\ ${ }^{17}$ Dipartimento di Fisica e Astronomia, Università di Padova, Vicolo dell'Osservatorio 3, Padova, I-35122, Italy \\ ${ }^{18}$ INAF-Osservatorio Astronomico di Padova, Vicolo dell'Osservatorio 5, Padova, I-35122, Italy \\ Received 2020 October 7; revised 2020 October 29; accepted 2020 October 30; published 2020 December 2
}

\begin{abstract}
We analyze the oxygen abundances of a stellar sample representative of the two major Galactic populations: the thin and thick disks. The aim is to investigate the differences between members of the Galactic disks and contribute to the understanding of the origin of oxygen chemical enrichment in the Galaxy. The analysis is based on the $[\mathrm{OI}]=6300.30 \AA$ oxygen line in high-resolution spectra $(R \sim 52,500)$ obtained from the Gaia-ESO public spectroscopic Survey (GES). By comparing the observed spectra with a theoretical data set computed in LTE with the SPECTRUM synthesis and ATLAS12 codes, we derive the oxygen abundances of 516 FGK dwarfs for which we have previously measured carbon abundances. Based on kinematic, chemical, and dynamical considerations, we identify 20 thin and 365 thick disk members. We study the potential trends of both subsamples in terms of their chemistry $([\mathrm{O} / \mathrm{H}],[\mathrm{O} / \mathrm{Fe}],[\mathrm{O} / \mathrm{Mg}]$, and $[\mathrm{C} / \mathrm{O}]$ versus $[\mathrm{Fe} / \mathrm{H}]$ and $[\mathrm{Mg} / \mathrm{H}])$, age, and position in the Galaxy. The main results are that (a) $[\mathrm{O} / \mathrm{H}]$ and $[\mathrm{O} / \mathrm{Fe}]$ ratios versus $[\mathrm{Fe} / \mathrm{H}]$ show systematic differences between thin and thick disk stars with an enhanced $\mathrm{O}$ abundance of thick disk stars with respect to thin disk members and a monotonic decrement of $[\mathrm{O} / \mathrm{Fe}]$ with increasing metallicity, even at metal-rich regime; (b) there is a smooth correlation of $[\mathrm{O} / \mathrm{Mg}]$ with age in both populations, suggesting that this abundance ratio can be a good proxy of stellar ages within the Milky Way; and (c) thin disk members with $[\mathrm{Fe} / \mathrm{H}] \simeq 0$ display a $[\mathrm{C} / \mathrm{O}]$ ratio smaller than the solar value, suggesting a possibly outward migration of the Sun from lower Galactocentric radii.
\end{abstract}

Unified Astronomy Thesaurus concepts: Late-type stars (909); Stellar abundances (1577); Stellar ages (1581); Milky Way disk (1050)

\section{Introduction}

Oxygen is the third most abundant element in the universe and is produced by hydrostatic burning in massive stars and then essentially mostly dispersed by Type II supernovae ( $\mathrm{SNe}$ II; Woosley \& Weaver 1995). Oxygen and its isotopes provide fundamental evolutionary information on a number of astrophysical topics from exoplanets to galaxies, including, of particular importance, studies of the chemical evolution of the Milky Way (Matteucci \& Chiappini 2001). Moreover, in recent years, numerous studies have been devoted to investigating whether or not there is a correlation between the stellar oxygen abundance and the presence of exoplanets (see Brewer \&

\footnotetext{
${ }^{*}$ Based on observations collected with the FLAMES instrument at the VLT/ UT2 telescope (Paranal Observatory, ESO, Chile) for the Gaia-ESO Large Public Spectroscopic Survey (188.B-3002, 193.B-0936).
}

Fischer 2016; Nissen \& Gustafsson 2018; Pavlenko et al. 2019).

Considerable efforts have been made in the literature in recent years in deriving an accurate $\mathrm{O}$ abundance in late-type stars. Unfortunately, the atomic lines suitable for deriving the $\mathrm{O}$ abundance are not numerous in the visual spectrum of FGK stars, and all of them present some difficult issues. The most common oxygen lines used in the literature are the $7773 \AA$ triplet lines $(\mathrm{OI}=7772,7774$, and $7775 \AA)$, the highexcitation O I $6158 \AA$ line, and the forbidden lines [O I] 6300 and $6363 \AA$. The O I triplet lines are relatively easily accessible and located in a spectral region not affected by blending lines, but they are particularly sensitive to 3D nonlocal thermodynamic equilibrium (NLTE; e.g., Asplund et al. 2009; Amarsi et al. 2015, 2016, 2019). On the other hand, the other lines, less problematic for NLTE effects, are quite weak and, therefore, difficult to measure at relatively low and high temperatures. An 
accurate analysis and study of these lines was performed by Bertran de Lis et al. (2015), who also discussed the influence of the quality and signal-to-noise ratio of the spectra on the discrepancies of results obtained according to the different lines used. Moreover, some of these lines can be severely contaminated by the presence of telluric lines.

A different approach uses the strengths of the large number of molecular lines of $\mathrm{OH}$ in the near-UV and near-IR (Israelian et al. 1998, 2001a, 2001b; Boesgaard et al. 1999a, 1999b; Meléndez et al. 2001) as an indicator of oxygen abundance. However, the $\mathrm{OH}$ lines, and thus the oxygen abundance derived from them, are very sensitive to the adopted effective temperature (Jönsson et al. 2018) and 3D granulation effects (González Hernández et al. 2010).

Since different oxygen diagnostics provide discordant results (see, for example, Bertran de Lis et al. 2015), the distribution of the $[\mathrm{O} / \mathrm{Fe}]$ abundance ratio in stars across the Galactic disk(s) is still under debate. Many papers in the literature are focused on the understanding of the discrepancies derived by the different lines adopted to estimate oxygen abundance and their effect on the analysis of oxygen trends in the disk populations of our Galaxy (e.g., Bensby et al. 2004; Bertran de Lis et al. 2015).

Nowadays, in addition to all of these numerous and accurate works, we also have the huge amount of data provided by large modern spectroscopic surveys, such as the Gaia-ESO public spectroscopic Survey (GES; Gilmore et al. 2012; Randich et al. 2013; ESO programs 188.B-3002 and 193.B-0936), the Apache Point Observatory Galactic Evolution Experiment (APOGEE; Majewski et al. 2017), and GALactic Archaeology with HERMES (GALAH; De Silva et al. 2015), that, by providing multielemental abundance ratios of thousands of stars belonging to different Galactic populations, constitute a wealth of valuable information for theoretical studies. In studying the chemical properties of stellar populations in the Galaxy, some of the above works have obtained discrepant results. For instance, assuming $\mathrm{Mg}$ instead of $\mathrm{Fe}$ as a reference element $(\mathrm{Mg}$ is thought to come mostly from core-collapse supernovae, CCSNe), Weinberg et al. (2019) analyzed a spectroscopic sample of 20,485 stars within the Sloan Digital Sky Survey APOGEE survey. They derived the median trends of abundance ratios $[\mathrm{X} / \mathrm{Mg}]$ versus $[\mathrm{Mg} / \mathrm{H}]$ for 15 elements and fit these trends with a simple "two-process" model that characterizes the relative production of CCSNe and SNe Ia. They found, for the particular case of oxygen, that $[\mathrm{O} / \mathrm{Mg}]$ barely correlates with $[\mathrm{Mg} / \mathrm{H}]$. Conversely, Griffith et al. (2019) found a strong correlation with $[\mathrm{O} / \mathrm{Mg}]$ values that significantly decrease with increasing $[\mathrm{Mg} / \mathrm{H}]$, a result in agreement with the work of Bensby et al. (2014). As mentioned above, inferring oxygen abundances from optical or near-IR spectra can be very challenging, principally due to NLTE corrections or the impact of $T_{\text {eff }}$ on $\mathrm{OH}$ molecular abundances, respectively.

In this paper, we take advantage of the GES to derive oxygen abundances for a sample of 516 FGK dwarf stars belonging to the Galactic disks. These stars are a subsample of a larger (2133 objects) set of dwarfs stars already analyzed by Franchini et al. (2020, hereafter FR20) to derive carbon abundances from atomic lines in their UVES spectra. In this paper, in order to derive oxygen abundances, we use the [O I] 6300.304 $\AA$ forbidden line, which is unaffected by NLTE effects, with the aim of constructing and analyzing the trends of $[\mathrm{O} / \mathrm{H}],[\mathrm{O} / \mathrm{Fe}]$,
$[\mathrm{O} / \mathrm{Mg}]$, and $[\mathrm{C} / \mathrm{O}]^{19}$ versus $[\mathrm{Fe} / \mathrm{H}],[\mathrm{Mg} / \mathrm{H}]$, and spatial position in the Galaxy and age for the thin and thick disk populations. Our final goal is to obtain detailed information on the chemical evolution, in particular of $[\mathrm{O} / \mathrm{Fe}]$ and $[\mathrm{C} / \mathrm{O}]$. The sample contains stars with $T_{\text {eff }}$ from 4877 to $6561 \mathrm{~K}$ and $[\mathrm{Fe} / \mathrm{H}]$ from -0.84 to +0.46 dex.

The paper is structured as follows. In Section 2, we introduce the sample used in this work. In Section 3, we describe the method adopted for the oxygen abundance determination and define the thin and thick disk samples by using the three selection criteria presented in FR20. Section 4.1 is dedicated to the discussion of the $[\mathrm{O} / \mathrm{Fe}]-[\mathrm{Fe} / \mathrm{H}]$ and $[\mathrm{O} / \mathrm{Mg}]-[\mathrm{Mg} / \mathrm{H}]$ trends for both the thin and thick disk samples, while Section 4.2 is dedicated to the $[\mathrm{C} / \mathrm{O}]$ trends with $[\mathrm{Fe} / \mathrm{H}]$, $[\mathrm{Mg} / \mathrm{H}]$, and $[\mathrm{O} / \mathrm{H}]$. We also discuss the $[\mathrm{O} / \mathrm{H}],[\mathrm{O} / \mathrm{Fe}],[\mathrm{O} /$ $\mathrm{Mg}$ ], and [C/O] trends with $R_{\text {med }}$ (the mean of the apo- and pericentric distances of the stellar orbit), with $\left|Z_{\max }\right|$ (the maximum distance from the Galaxy plane achieved by a star during its orbit; Section 4.3) and age (Section 4.4). A comparison of our results with those from other surveys is presented in Section 5. Conclusions are given in Section 6.

\section{Observational Data}

For the aim of our work, we started from the sample of 2133 observed stars extracted from the fifth GES internal data release (GES iDR5) and whose carbon abundances were derived from atomic lines in FR20. The sample (hereafter the UVES-U580 sample) contains spectra of FGK dwarf stars obtained with the UVES spectrograph in the setup centered at $5800 \AA$. These spectra are exposed onto two CCDs, resulting in wavelength coverage of $4700-6840 \AA$ with a gap of $\sim 50 \AA$ in the center and spectral resolving power $\frac{\lambda}{\Delta \lambda} \sim 47,000$ and $\sim 52,000^{20}$ in the blue and red parts of the spectra, respectively. For the sake of easy reference, here we only mention the main properties of our sample. The reader is referred to FR20 for details about the criteria adopted to select the GES spectra.

The UVES-U580 sample consists of dwarfs stars with homogeneously determined GES effective temperatures ( $\left.T_{\text {eff }}\right)$, surface gravities $(\log g)$, iron abundances $([\mathrm{Fe} / \mathrm{H}])$, microturbulence $(\xi)$, radial velocities $\left(v_{\mathrm{r}}\right)$, rotational velocities $(v \sin i)$, and detailed chemical compositions ${ }^{21}$ spanning the following ranges: $T_{\text {eff }}$ from 4599 to $6868 \mathrm{~K}, \log g$ from 3.50 to $4.79 \mathrm{dex},[\mathrm{Fe} / \mathrm{H}]$ from -0.90 to $+0.60 \mathrm{dex}$, and $[\mathrm{C} / \mathrm{Fe}]$ from -0.44 to +0.44 dex. In FR20, the sample was restricted to only dwarf and turnoff stars in order to avoid the stellar evolution effects in the red giants, where, during the first dredge-up phase, their original CNO atmosphere composition is altered (Iben 1964, 1967; Becker \& Iben 1979).

The availability of accurate parallaxes and proper motions for a large fraction of stars in our sample ${ }^{22}$ from the second Gaia data release (DR2; Gaia Collaboration et al. 2018) and radial velocities from the GES iDR5 allowed FR20 to compute Galactic velocities, orbits, and absolute magnitudes for 1804 dwarfs, together with derived Bayesian ages for 1751 stars.

\footnotetext{
${ }^{19}$ Iron and magnesium abundances are from the GES iDR5, and carbon abundances are from FR20.

${ }^{20}$ According to the SPEC_RES keyword in the primary header of the observed spectra.

${ }^{21}$ The GES abundances of element $X$ are given as $\log \epsilon_{X}=\log \frac{N_{\mathrm{X}}}{N_{\mathrm{H}}}+12.0$.

${ }^{22} \mathrm{We}$ considered only stars with small relative errors, i.e., less than $10 \%$ in parallaxes and proper motions.
} 


\section{Oxygen Abundances}

The GES spectra do not cover the O I 7771-3 $\AA$ triplet lines but incorporate the OI $6158 \AA$ permitted line and forbidden [O I] 6300 and $6363 \AA$ lines. Since the crowding of spectral features surrounding the stellar O I $6158 \AA$ line makes the required normalization to the continuum a very challenging process, and because of the intrinsic weakness of the feature, after a detailed inspection of the spectra, we decided not to use this line. The forbidden [O I] $6363 \AA$ line is also very weak in our spectra and not suitable to derive reliable abundances. So, we were compelled to restrict our oxygen abundance determination to only the strongest forbidden line of oxygen at $6300 \AA$.

The [OI] line at $6300 \AA$ is blended with the $\mathrm{Ni}$ line at $6300.336 \AA$ (see top panel of Figure 1 and Bensby et al. 2004); therefore, the determination of oxygen abundance via this line implies knowledge of the Ni abundance. Fortunately, the GES iDR5 provides the Ni abundance for all of the stars of our sample. In order to properly account for the Ni contamination, and considering that the line is usually very weak, we have used the very high quality spectrum of the Sun used in FR20 to fine-tune the $\log g f$ values of all of the lines needed to satisfactorily reproduce the [O I] and $\mathrm{Ni}$ I line blend in the solar spectrum (see top panel of Figure 1). The adopted astrophysical $\log g f$, derived as described in Franchini et al. (2018), are reported in Table 1, together with values from the literature; we point out that we have used the same $\log g f$ value $(-2.21)$ for the two isotopic components of the Ni line (see Johansson et al. 2003) and an isotopic abundance ratio $\frac{\mathrm{Ni}^{58}}{\mathrm{Ni}^{60}}=2.6$.

It is important to also note that, depending on the stellar radial velocity and heliocentric velocities of the observations, the [O I] line at $6300 \AA$ can be blended, sometimes severely, with telluric absorption lines as sketched in the middle panel of Figure 1 and affected, in some spectra, by poor removal of the night-sky $[\mathrm{OI}]=6300.30 \AA$ emission line (Burnside et al. 1977; Nissen \& Edvardsson 1992; Osterbrock et al. 1996; see the bottom panel of Figure 1). Therefore, particular care was adopted to check and reject the spectra where the [O I] line profile was affected by the abovementioned problems (see Section 3.2).

\subsection{Atmosphere Models and Synthetic Spectra}

To estimate $[\mathrm{O} / \mathrm{Fe}]$ ratio abundances, we adopted a spectral synthesis technique. For each of the 2133 stars, we used the stellar atmosphere ATLAS12 code (Kurucz 2005) and the spectral synthesis program SPECTRUM v2.76f (Gray \& Corbally 1994) to compute its model atmosphere and synthetic spectrum, respectively. As described in FR20, we adopted ATLAS12, since it allows us to generate on-the-fly models with full consistency between the chemical composition used to build the atmosphere structure and the one actually used in synthesizing the emergent spectrum. In particular, for each $i$ th star, we used its GES iDR5 atmospheric parameter values ( $T_{\text {eff }}$, $\log g,[\mathrm{Fe} / \mathrm{H}]$, and $\xi)$ and individual element abundances with the only exception of $[\mathrm{C} / \mathrm{Fe}]$ values, which are from FR20 (for those elements with no estimate of $[\mathrm{X} / \mathrm{Fe}]$ we assumed $[\mathrm{X} /$ $\mathrm{Fe}]=0)$ to compute 13 atmosphere models differing only in $[\mathrm{O} / \mathrm{Fe}]$, i.e., with $[\mathrm{O} / \mathrm{Fe}]_{j}=-0.6+(j-1) \times 0.1 \mathrm{dex}$ (with $j=1, \ldots, 13)$.

Then, starting from each $i, j$ ( $i$ and $j$ specify the star and the adopted $[\mathrm{O} / \mathrm{Fe}]$ ratio, respectively) atmosphere model, we used
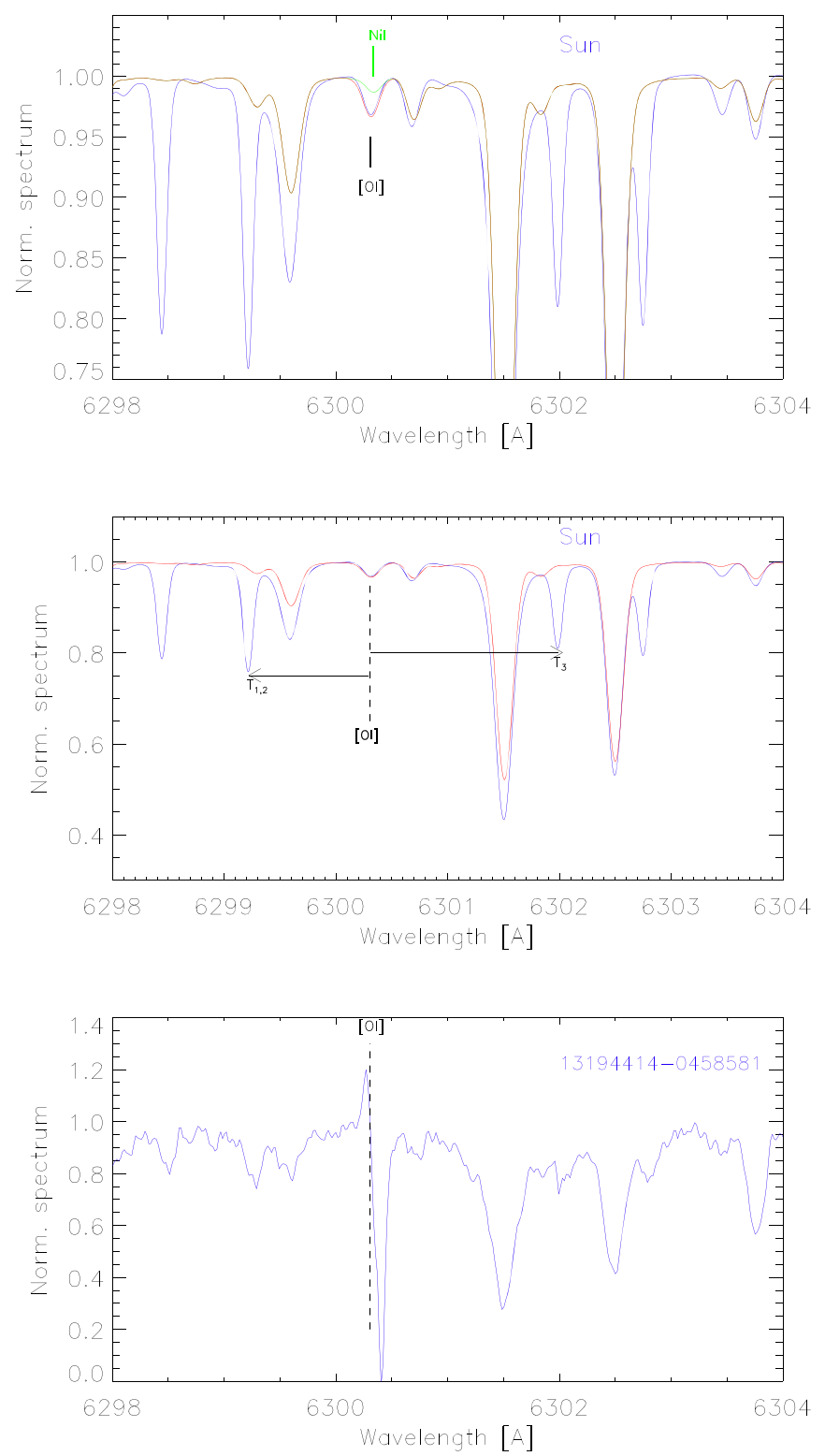

Figure 1. The top panel shows the contribution of $\mathrm{Ni} I$ lines to the formation of the $6300.3 \AA$ blend in the solar spectrum: observed solar spectrum (blue) and synthetic spectra with synthesized [O I] and $\mathrm{Ni}$ I lines (red) or without the [O I] line (green). The middle panel shows the comparison of synthetic (red) and observed (blue) solar spectra, where strong observed features not present in the synthetic spectrum correspond to telluric lines; e.g., $\mathrm{T}_{1,2}$ and $\mathrm{T}_{3}$ indicate the $6299.214 \mathrm{O}_{2}, 6299.227 \mathrm{H}_{2} 0$, and $6301.985 \mathrm{O}_{2}$ lines. Horizontal lines show the shifts of an observed stellar spectrum that would bring the telluric lines $T_{1,2}$ or $\mathrm{T}_{3}$ to overlap the [O I] feature; the corresponding velocity offsets due to the combined effect of stellar radial velocity and heliocentric velocity of the observation are -39 and $+92 \mathrm{~km} \mathrm{~s}^{-1}$, respectively. The bottom panel shows an example of GES UVES-U580 spectra with a residual of the removal of the night-sky $[\mathrm{O}$ I $]=6300.30$ emission line contaminating the $[\mathrm{O} \mathrm{I}]$ stellar feature.

Table 1

Oscillator Strength $g f$ of $[\mathrm{O}$ I] and Ni I Lines from Various Sources

\begin{tabular}{lccl}
\hline \hline $\begin{array}{l}\text { Line } \\
(\AA)\end{array}$ & [O I] & Ni I & References \\
\hline & -9300.304 & 6300.336 & \\
& -9.817 & -2.310 & 3D; Allende Prieto et al. (2001) \\
$\log g f$ & -9.717 & -2.135 & 1D; Bensby et al. (2004) \\
& -9.717 & -2.602 & 1D; Bertran de Lis et al. (2015) \\
& -9.715 & -2.210 & 1D; Pavlenko et al. (2019) \\
\end{tabular}


SPECTRUM v2.76f to obtain the corresponding normalized spectrum $\left(S_{\mathrm{N}}^{i, j}\right)$ in LTE approximation. The line list of atomic and molecular transitions we used in computing the synthetic spectra is the INTRIGOSS (high-resolution synthetic spectral library; Franchini et al. 2018) ${ }^{23}$ line list, updated by the new $\log g f$ values of Table 1 in FR20 and extended, by using the same technique described in Franchini et al. (2018), to also cover the wavelength range 6280-6325 A. For the reference solar abundances, we adopted those obtained by Grevesse et al. (2007).

In conclusion, for each star, we computed a set of 13 normalized synthetic spectra with $[\mathrm{O} / \mathrm{Fe}]$ consistent with the abovementioned atmosphere models. Since the synthetic spectra were computed at a very high resolving power ( 240,000), they were broadened by using the GES iDR5 $v \sin i$ stellar values and degraded at the resolution of red UVES spectra $(R \sim 52,500)$.

In order to remove the instrumental signature in the $[\mathrm{O} I]=6300 \AA$ line region of the observed (stacked) UVESU580 spectra, for each star $i$, we used the $j$ normalized synthetic $S_{\mathrm{N}}^{i, j}$ spectra to obtain a set of 13 normalized observed spectra $\left(\mathrm{O}_{\mathrm{N}}^{i, j}\right)$ from the corresponding observed UVES-U580 spectrum. The normalization was performed by applying the technique described in Franchini et al. $(2018,2020)$ but only in the region surrounding the stellar [O I] $6300 \AA$ line. Actually, we extracted the 6280-6325 $\AA$ wavelength region from the observed spectrum; searched for quasi-continuum flux reference points in $S_{\mathrm{N}}^{i, j}$ (i.e., wavelength points with flux levels in excess of 0.97), taking care to exclude regions affected by telluric features in the observed spectrum; and used the same points in the corresponding observed UVES spectrum to derive the continuum shape via a linear fitting of the ratio between observed and synthetic spectra. Eventually, the observed spectrum is divided by the computed linear fit to obtain the normalized spectrum $\mathrm{O}_{\mathrm{N}}^{i, j}$.

\section{2. [O/Fe] Determination: Synthesis of the [O I] $6300 \AA$ Line}

For each $i$ star and $j$ pair of spectra, i.e., for different $[\mathrm{O} / \mathrm{Fe}]$ values, we computed the standard deviation $\left(\sigma_{j}^{i}\right)$ between $\mathrm{O}_{\mathrm{N}}^{i, j}$ and $S_{\mathrm{N}}^{i, j}$ in a wavelength region centered at $6300.3038 \AA$ and with a width proportional to the stellar rotational velocity $(v \sin i)$, taking into account its uncertainty $\left(\epsilon_{v \sin i}\right.$; i.e., $\lambda_{0} \pm \Delta \lambda_{\text {rot }}$, where $\lambda_{0}=6300.3038 \AA, \Delta \lambda_{\text {rot }}=\left(v \sin i+\epsilon_{v \sin i}\right) \lambda_{0} / c$, and $c$ is the speed of light). The above process was implemented after we checked for the presence of

1. a badly removed night-sky $[\mathrm{O} \mathrm{I}]=6300.30 \AA$ emission line in the normalized spectra, and all spectra showing points with normalized flux values greater than 1.05 in the spectral range defined for computing $\sigma_{j}^{i}$ were rejected; and

2. contaminating telluric lines by rejecting all spectra with regions 1.5 times below the minimum predicted by all $\mathrm{S}_{\mathrm{N}}^{i, j}$ spectra in the spectral range defined for computing $\sigma_{j}^{i}$.

Then, using a parabolic fitting, we determined the "best" [O/ $\mathrm{Fe}$ ] value corresponding to the position of the minimum (if any) of $\sigma_{j}^{i}$ versus [O/Fe] (see FR20 for details, top panels of their Figure 2). In such a way, we were able to obtain $[\mathrm{O} / \mathrm{Fe}]$ estimates for 869 stars (no clear minimum in $\sigma_{j}^{i}$ was detected

\footnotetext{
${ }^{23}$ http://archives.ia2.inaf.it/intrigoss/
}

for the other stars, thus preventing a sound determination of $[\mathrm{O} / \mathrm{Fe}])$. We assumed as the uncertainty in the obtained $[\mathrm{O} / \mathrm{Fe}]$ the half-step of our $[\mathrm{O} / \mathrm{Fe}]$ grid of models and synthetic spectra, i.e., $\sigma_{[\mathrm{O} / \mathrm{Fe}]} \pm 0.05 \mathrm{dex}$.

To evaluate the variation of the oxygen abundance determinations when the uncertainties in the adopted model atmosphere parameters are taken into account, we rederived $[\mathrm{O} / \mathrm{Fe}]$ for some representative stars by varying their input $T_{\text {eff }}$, $\log g$, and $[\mathrm{Fe} / \mathrm{H}]$ values by plus or minus their typical uncertainties, which, for our sample, are $60 \mathrm{~K}, 0.1 \mathrm{dex}$, and 0.1 dex, respectively. We choose two pairs of relatively cool and hot stars, one with $[\mathrm{O} / \mathrm{Fe}] \simeq 0$ and one with $[\mathrm{O} /$ $\mathrm{Fe}]>0.3$ dex. In any case, the new $[\mathrm{O} / \mathrm{Fe}]$ determinations differ from the nominal ones by less than $0.05 \mathrm{dex}$ and indicate that the most critical parameter is $\log g$. Furthermore, to avoid any systematic error due to the use of a possible incorrect $\mathrm{Ni}$ abundance in evaluating the blend contribution of the $\mathrm{Ni}$ lines, we decided to reapply our fitting procedure to only half of the [O I] profile (i.e., the blue wing of the line profile, since the blend effect is much stronger in the red part of the [O I] line profile, as shown in the top panel of Figure 1) and to compare the obtained $[\mathrm{O} / \mathrm{Fe}]$ with that derived from the whole profile. Eventually, we rejected all stars/spectra with a standard deviation of the two obtained $[\mathrm{O} / \mathrm{Fe}]$ values greater than $3 \sigma_{[\mathrm{O} / \mathrm{Fe}]}$, thus obtaining a final sample of 516 dwarf stars with trustworthy $[\mathrm{O} / \mathrm{Fe}]$ abundance ratios (the adopted values are those from the fit of the whole profile). Moreover, to assess the absence of any other systematic offset in the derived $[\mathrm{O} / \mathrm{Fe}]$ values, we also applied the above-described procedure to the solar spectrum, obtaining $[\mathrm{O} / \mathrm{Fe}]_{\odot}=-0.02$.

The comparison of our results for oxygen abundance determination with those available in the literature can, unfortunately, only be done for very few of our stars. In fact, we found only 3, 4, and 10 stars in common with Bensby et al. (2014), Brewer \& Fischer (2016), and Buder et al. (2019), respectively. In any case, our results are in reasonable agreement with those obtained in these three works, taking into account that different oxygen lines and NLTE corrections were used (we recall that our results do not require any NLTE correction, since we used the [O I] 6300 line). Actually, we found mean differences in [O/ $\mathrm{H}]$ of $0.06 \pm 0.08,-0.24 \pm 0.10$, and $0.09 \pm 0.12$ for the stars in common with Bensby et al. (2014), Brewer \& Fischer (2016), and Buder et al. (2019), respectively, where the highest difference is very likely due to the absence of any NLTE correction in Brewer \& Fischer (2016).

Out of the 516 stars, 308 also have an age determination (for the other 208 stars, FR20 found too-uncertain ages, i.e., with FWHM $_{\text {age }}>8$ Gyr).

Figure 2 shows the histograms of the atmospheric parameters and FR20 ages for the stars in the final sample. The final sample consists of stars spanning $T_{\text {eff }}$ from 4877 to $6561 \mathrm{~K}$, $\log g$ from 3.51 to $4.79 \mathrm{dex}$, and $[\mathrm{Fe} / \mathrm{H}]$ from -0.84 to 0.46 dex.

\subsection{Definition of Thin and Thick Disk Star Samples}

To discriminate between thin and thick disk stars in the UVES-U580 sample, we used, as in FR20, three different selection methodologies: (i) the chemical one based on positions in the $[\mathrm{Mg} / \mathrm{Fe}]-[\mathrm{Fe} / \mathrm{H}]$ plane, (ii) the kinematical one based on stellar Galactic velocities, and (iii) the orbital one based on the $R_{\text {med }}$ and $\left|Z_{\text {max }}\right|$ orbital parameters (see FR20 for details). As already discussed in FR20 (and references therein), 

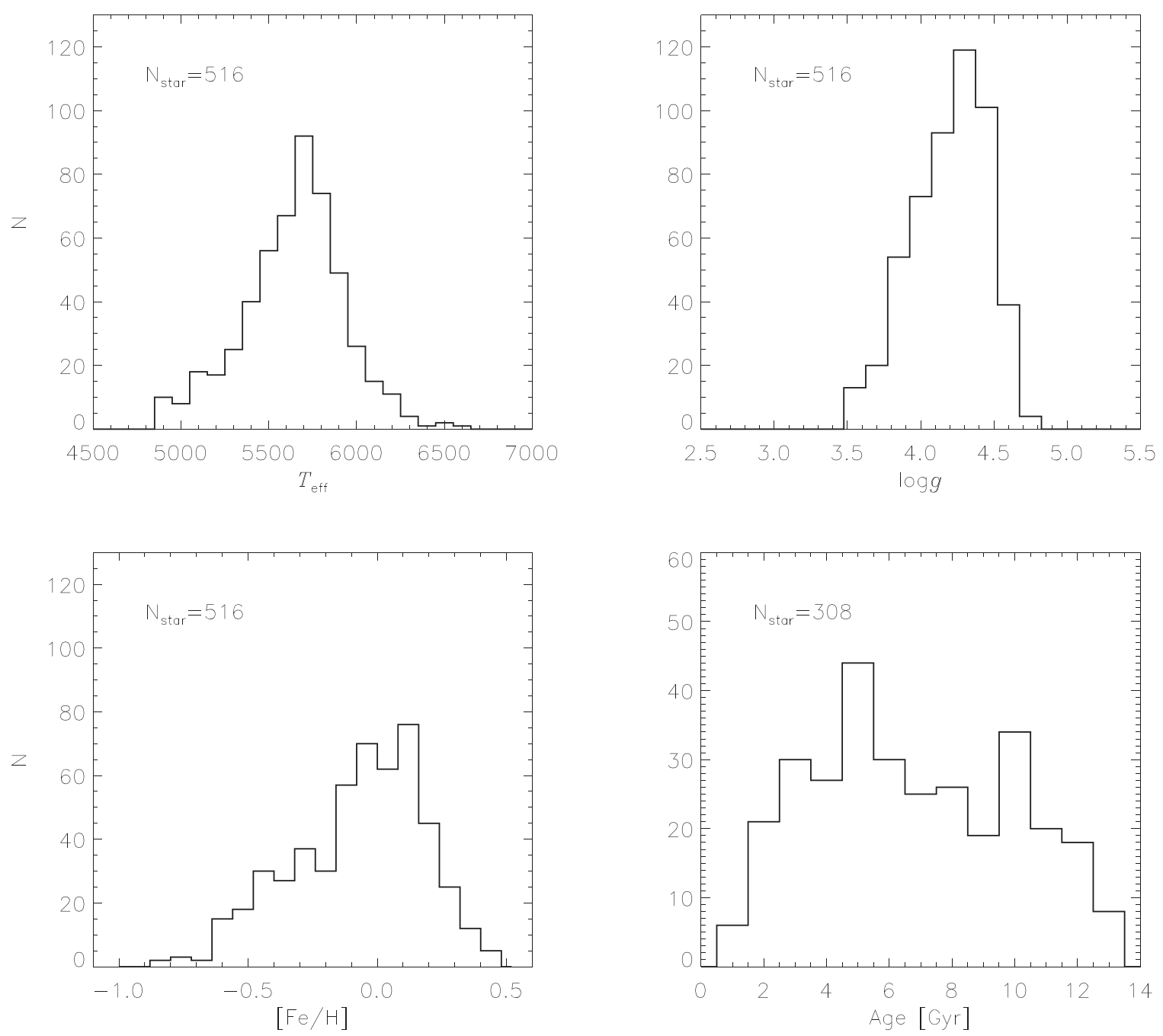

Figure 2. Histograms of atmospheric parameter values for the 516 stars with trustworthy $[\mathrm{O} / \mathrm{Fe}]: T_{\text {eff }}$ (top left panel), log $g$ (top right panel), [Fe/H] (bottom left panel), and age (bottom right panel) for the subset of 308 stars (see text).

there are no conclusive criteria yet for unambiguously separating samples of thin and thick disk stars with null contamination. In fact, depending on the adopted criterion, the number of classified (i.e., as thin or thick disk stars) and unclassified stars in our sample actually varies. In fact, even if thin and thick disk stars should differ in both chemistry and kinematics, the sensitivity of each above-listed classification method varies for different kinds of stars. In particular,

1. the adopted chemical classification, taking into account the uncertainties on $[\mathrm{Mg} / \mathrm{Fe}]$ and $[\mathrm{Fe} / \mathrm{H}]$ determinations, is less effective for metal-rich stars due to the merging of the two sequences of high- and low- $\alpha$ objects at $[\mathrm{Fe} / \mathrm{H}]>0$

2. the kinematical classification, also affected by uncertainties in the computed Galactic velocities, has difficulty classifying objects that fall in the overlap regions of the
Gaussian velocity distributions of the different Galactic components; and

3. the classification based on the orbital parameters $R_{\text {med }}$ and $\left|Z_{\max }\right|$, whose computed values depend not only on the accuracy of the input stellar positions, distances, and velocities but also on the reliability of the adopted Galactic potential, may fail for stars falling close to the somewhat arbitrary separation borders in the orbital parameter plane between the different Galactic components adopted in FR20.

On the other hand, FR20 showed that, independent of the classification method adopted, in all thin and thick disk star subsamples, the behaviors of $[\mathrm{C} / \mathrm{H}],[\mathrm{C} / \mathrm{Mg}]$, and $[\mathrm{C} / \mathrm{Fe}]$ versus $[\mathrm{Fe} / \mathrm{H}],[\mathrm{Mg} / \mathrm{H}]$, and age and the Galactic regions they populate (given by the stellar orbit $R_{\text {med }}$ and $\left|Z_{\max }\right|$ ) are very similar. Therefore, in this paper, we decided to merge the results of the three abovementioned selection methodologies in the following way:

$$
\begin{aligned}
& \text { Thin-disk star if } \quad\left(\text { Thin }^{\mathrm{C}}=\text { true and/or } \operatorname{Thin}^{\mathrm{K}}=\text { true and/or Thin }{ }^{\mathrm{O}}=\text { true }\right) \text { and } \\
& \text { (Thick }{ }^{\mathrm{C}}=\text { false and } \text { Thick }^{\mathrm{K}}=\text { false and Thick }{ }^{\mathrm{O}}=\text { false) } \\
& \text { Thick-disk star if }\left(\text { Thick }^{\mathrm{C}}=\text { true and } / \text { or Thick }^{\mathrm{K}}=\text { true and/or Thick }{ }^{\mathrm{O}}=\text { true }\right) \text { and } \\
& \left(\text { Thin }{ }^{\mathrm{C}}=\text { false and } \operatorname{Thin}^{\mathrm{K}}=\text { false and } \operatorname{Thin}^{\mathrm{O}}=\right.\text { false), }
\end{aligned}
$$



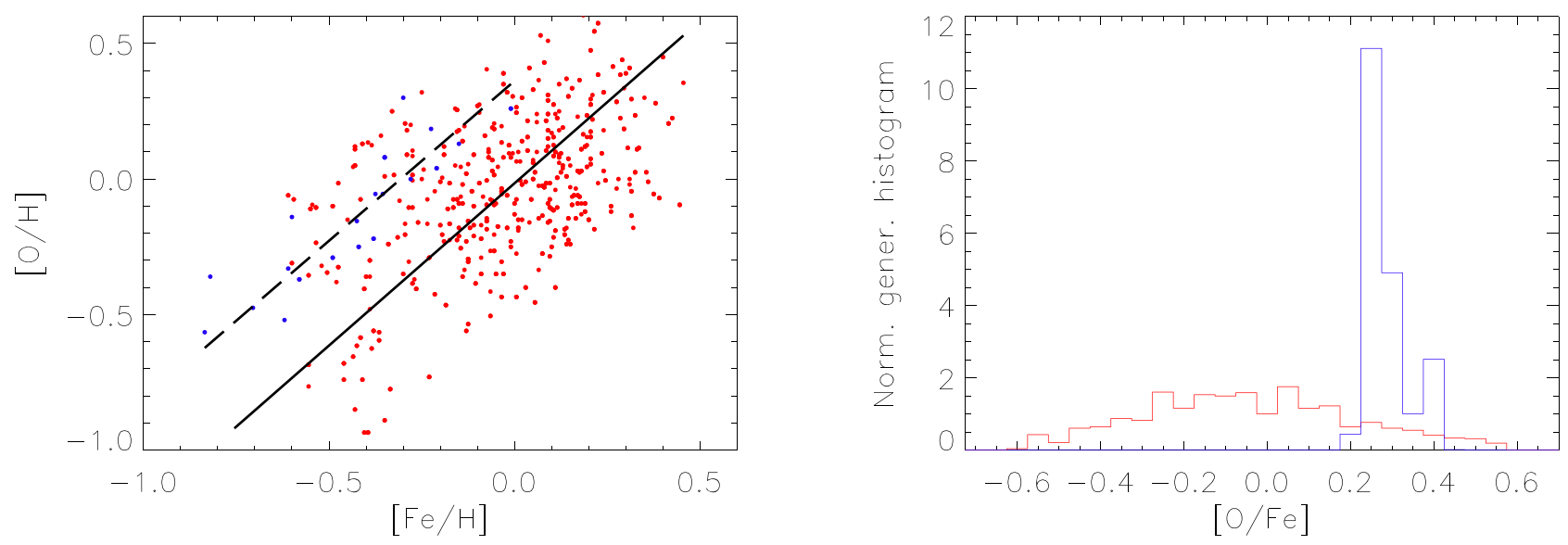

Figure 3. The $[\mathrm{O} / \mathrm{H}]-[\mathrm{Fe} / \mathrm{H}]$ diagrams (left panel) and $[\mathrm{O} / \mathrm{Fe}]$-normalized generalized histograms (right panel) for thin (red) and thick (blue) disk star samples. Regression lines for thin (solid) and thick (dashed) disk stars are superimposed on the left panel. Oxygen abundances are from this paper, while $[\mathrm{Fe} / \mathrm{H}]$ is from the GES iDR5.

where the superscripts "C," "K," and "O" refer to the results of the chemical, kinematical, and orbital methodology, respectively. In such a way, after discarding the few stars with discordant classification, we obtained two samples of 376 and 20 stars classified by at least one methodology as thin or thick disk stars, respectively, even if they were unclassified by the other methodologies. In such a way, we tried to also deal with the uncertainties on the different parameters used for the different classification approaches. We recognize that the thick disk sample is small; therefore, it is desirable to collect more data for this population to confirm the robustness of the results presented in the following sections.

\section{Oxygen Trends in the Thin and Thick Disks}

The left panel of Figure 3 shows the $[\mathrm{O} / \mathrm{H}]$ versus $[\mathrm{Fe} / \mathrm{H}]$ for the thin (red points) and thick (blue points) disk sample stars, together with their regression lines (solid and dashed lines for thin and thick disk samples, respectively). Even if the thick disk sample does not contain a very large number of stars, we can observe that, in general, the thick disk stars have a larger $\mathrm{O}$ abundance, by about $0.35 \mathrm{dex}$, than the thin disk stars at the same $[\mathrm{Fe} / \mathrm{H}]$. The slopes of the regression lines do not differ significantly, as can be seen in Table 2 , where the slope $(s)$ and intercept $(i)$ coefficients with their standard deviations $\left(\sigma_{s}\right.$ and $\sigma_{i}$, respectively) are reported. In the right panel of Figure 3, we show the normalized generalized distributions of $[\mathrm{O} / \mathrm{Fe}]$ built by summing the individual unit area Gaussian computed for each star by using its $[\mathrm{O} / \mathrm{Fe}]$ value and uncertainty and normalizing the results to the number of entries. In computing the regression lines and building the normalized distributions, we weighted each star one, two, or three times according to the number of methodologies that lead to its classification. The thin disk sample distribution (red) of $[\mathrm{O} / \mathrm{Fe}]$ is quite broad and has its maximum at $[\mathrm{O} / \mathrm{Fe}] \approx 0.0 \mathrm{dex}$, while the thick disk sample distribution (blue) is much narrower and peaks at $[\mathrm{O} / \mathrm{Fe}] \approx$ 0.25 dex, being consistent with a much faster and more efficient formation of the thick disk.

Figure 3 also shows that both the thin and thick disk stars display a large scatter in the $[\mathrm{O} / \mathrm{H}]$ values. Such a scatter for this abundance ratio was also found (and discussed) by several authors (e.g., Bertran de Lis et al. 2015, 2016; Nissen \& Gustafsson 2018; Amarsi et al. 2019), as well as for other chemical elements in Galactic abundance trends, however, to a
Table 2

Linear Regression Coefficients

\begin{tabular}{lcccc}
\hline \hline & $s \pm \sigma_{s}$ & $i \pm \sigma_{i}$ & $N_{\text {star }}$ & $\chi_{\mathrm{r}}$ \\
\hline Thin & $1.20 \pm 0.03$ & $-0.02 \pm 0.01$ & 376 & 2.9 \\
Thick & $1.18 \pm 0.11$ & $0.36 \pm 0.05$ & 20 & 3.5
\end{tabular}

Note. Here $N_{\text {star }}$ is the number of points used to derive the regression lines.

lesser extent (Adibekyan et al. 2012). Since the above works and ours are based on different oxygen features, the results cannot be easily compared. The quite large vertical extension at a given $[\mathrm{Fe} / \mathrm{H}]$ might represent a truly cosmic scatter (e.g., Petigura \& Marcy 2011; Bertran de Lis et al. 2016) or its combination with uncertainties in our $[\mathrm{O} / \mathrm{Fe}]$ determinations. We nevertheless want to remark that if the scatter of the number of points of the fit, np, was purely statistical, we would expect our fits to have a reduced $\chi_{\mathrm{r}}=\sqrt{\frac{\chi^{2}}{\mathrm{np}-2}} \lesssim 1$, but our fits have reduced $\chi_{\mathrm{r}} \sim 3$ (see Table 2 ), which suggests that a significant part of the observed scatter is astrophysical.

\section{1. $[\mathrm{O} / \mathrm{Fe}]-[\mathrm{Fe} / \mathrm{H}]$ and $[\mathrm{O} / \mathrm{Mg}]-[\mathrm{Mg} / \mathrm{H}]$ Trends}

As mentioned earlier, several studies of the Galactic chemical history make use of $\mathrm{Mg}$ instead of the more popular and accessible $\mathrm{Fe}$ as a reference element. The choice is based on the potential single origin (SNe II) of $\mathrm{Mg}$ (e.g., Bensby et al. 2004; Griffith et al. 2019; Weinberg et al. 2019), although some stellar yield models have provided theoretical evidence that $\mathrm{Mg}$ might also be partially released into the interstellar medium by SNe Ia (Magrini et al. 2017; Naiman et al. 2018). In this context, Bensby et al. (2004) recommended being very cautious about promoting one specific reference element, and they instead suggested considering more than one. Following their suggestion, in what follows, we analyze disk stellar populations on the basis of the abundance ratios $[\mathrm{O} / \mathrm{Fe}]$ and $[\mathrm{O} / \mathrm{Mg}]$ in terms of $[\mathrm{Fe} / \mathrm{H}]$ and $[\mathrm{Mg} / \mathrm{H}]$, respectively. The trends depicted in Figure 4 are computed using a running average (using bins with 100 points partially overlapped by shifting them by 10 points and bins with 10 points partially overlapped by shifting them by two points for the thin and thick disk stars, respectively), while the horizontal and vertical bars correspond to the standard deviations of the bin averages. 

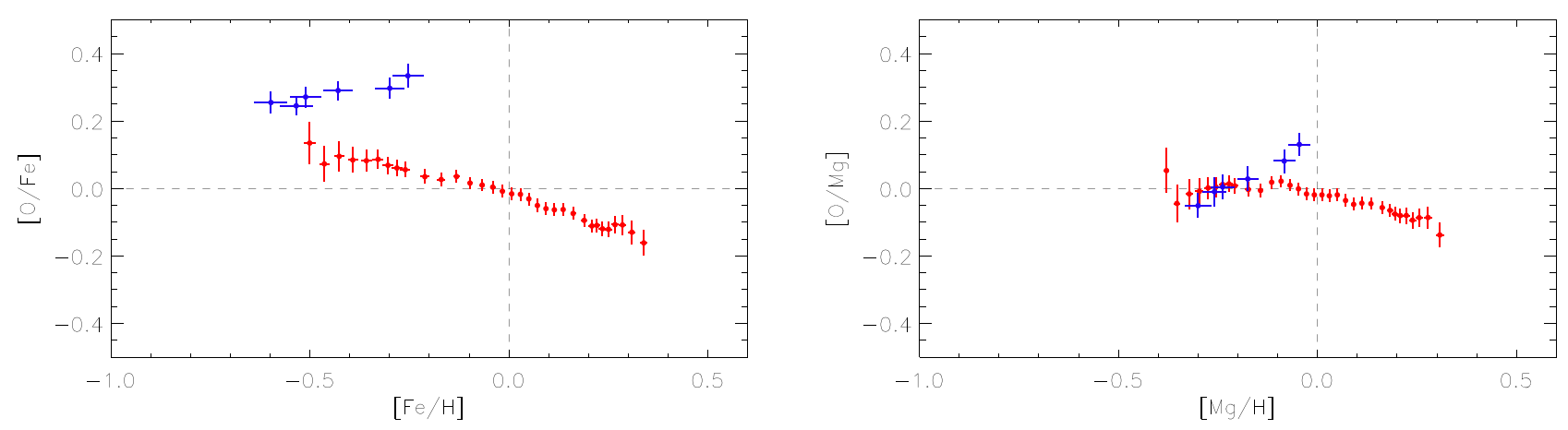

Figure 4. The $[\mathrm{O} / \mathrm{Fe}]-[\mathrm{Fe} / \mathrm{H}]$ diagrams (left panel) and $[\mathrm{O} / \mathrm{Mg}]-[\mathrm{Mg} / \mathrm{H}]$ diagrams (right panel) for thin (red) and thick (blue) disk star samples; binned running averages and standard deviations are plotted. Oxygen abundances are from this paper, while $[\mathrm{Fe} / \mathrm{H}]$ and $[\mathrm{Mg} / \mathrm{H}]$ are from the GES iDR5.

For the $[\mathrm{O} / \mathrm{Fe}]-[\mathrm{Fe} / \mathrm{H}]$ tendencies illustrated in the left panel of Figure 4, we distinguish the following properties.

1. We found systematic differences between thin and thick disk stars, as already hinted at by the distributions in Figure 3; thick disk stars show a higher $[\mathrm{O} / \mathrm{Fe}]$ than objects in the thin disk in the common $[\mathrm{Fe} / \mathrm{H}]$ interval. Thick disk stars show an almost flat distribution in the limited $[\mathrm{Fe} / \mathrm{H}]$ range they cover, while thin disk stars show a clear monotonic decrease of $[\mathrm{O} / \mathrm{Fe}]$ for increasing metallicity. As expected, these $[\mathrm{O} / \mathrm{Fe}]$ versus $[\mathrm{Fe} / \mathrm{H}]$ trends resemble the behavior of other $\alpha$-elements versus $[\mathrm{Fe} / \mathrm{H}]$. Actually, oxygen being an $\alpha$-element, for the most metal-poor stars in our sample, an $[\mathrm{O} / \mathrm{Fe}]$ plateau reflects a steady rate of oxygen and iron enrichment of the interstellar medium by CCSNe. The gradual decrease of $[\mathrm{O} / \mathrm{Fe}]$ with increasing $[\mathrm{Fe} / \mathrm{H}]$ reflects the iron enrichment from $\mathrm{SNe}$ Ia, which have a delayed distribution in time relative to CCSNe (e.g., Carigi et al. 2005; Maoz et al. 2012). Analogous systematic differences between thick and thin disk abundance distributions have been obtained in several works in the literature (e.g., Bensby et al. 2004, 2014; Reddy et al. 2006; Ramírez et al. 2013; Nissen et al. 2014; Bertran de Lis et al. 2015; Delgado Mena et al. 2019), all confirming that oxygen behaves like $\mathrm{Mg}$ and the other $\alpha$-elements but at high metallicity.

2. We can notice that at $[\mathrm{Fe} / \mathrm{H}] \approx-0.40$, there is a difference of $\Delta[\mathrm{O} / \mathrm{Fe}] \sim+0.2$ dex between the thin and thick disk star trends. Interestingly, Reddy et al. (2006) found, on the basis of a kinematical selection, that their thick and thin disk samples are also well separated. In their bin centered at $[\mathrm{Fe} / \mathrm{H}]=-0.50$, the mean abundance ratios $[\mathrm{O} / \mathrm{Fe}]$ are $0.36 \pm 0.19$ and $0.24 \pm$ 0.07 dex for the thick and thin disks, respectively. These values are in agreement with our results within the uncertainties.

In this context, the more recent work of Nissen et al. (2014) found that their thin disk stars fall well below the thick disk counterparts in the $[\mathrm{O} / \mathrm{Fe}]$ versus $[\mathrm{Fe} / \mathrm{H}]$ diagram by as much as $[\mathrm{Fe} / \mathrm{H}] \sim-0.3$, and they suggested that the two populations merge at higher metallicities. This later feature cannot be corroborated with our data, since we lack thick disk stars with metallicities higher than -0.2 dex.

3. At supersolar metallicity $([\mathrm{Fe} / \mathrm{H}]>0)$, we observe that the $[\mathrm{O} / \mathrm{Fe}]$ of thin disk stars continues to decrease, as also found by several authors (e.g., Castro et al. 1997; Chen et al. 2003; Bensby et al. 2004, 2014; Ecuvillon et al. 2006; Amarsi et al. 2019) in concordance with the prediction of Galactic chemical evolution models (e.g., Chiappini et al. 2003). In contrast, other works have found a flattening of the trend, i.e., a constant value of $[\mathrm{O} / \mathrm{Fe}] \sim 0$ (e.g., Nissen \& Edvardsson 1992; Nissen et al. 2002; Ramírez et al. 2013; Bertran de Lis et al. 2015). Our results suggest that the oxygen is produced only by CCSNe with no evidence of any SN Ia or asymptotic giant branch (AGB) star contribution that would produce a flattening of $[\mathrm{O} / \mathrm{Fe}]$ at $[\mathrm{Fe} / \mathrm{H}] \simeq 0$, as observed in other $\alpha$-elements (Bensby et al. 2004, 2014). We notice that the thin disk star $[\mathrm{O} / \mathrm{Fe}]$ trend continues down to an underabundance of $[\mathrm{O} / \mathrm{Fe}] \approx-0.15$ at $[\mathrm{Fe} /$ $\mathrm{H}] \approx 0.30$ in agreement with the results from Bensby et al. (2004), who, adopting a kinematic selection for their thin disk sample, showed a decreasing trend leading to $[\mathrm{O} / \mathrm{Fe}] \approx-0.2$ at $[\mathrm{Fe} / \mathrm{H}] \approx 0.4 \mathrm{dex}$.

4. The linear relation between $[\mathrm{O} / \mathrm{Fe}]$ and $[\mathrm{Fe} / \mathrm{H}]$ for the thin disk stars,

$[\mathrm{O} / \mathrm{Fe}]=(-0.33 \pm 0.01) \times[\mathrm{Fe} / \mathrm{H}]-(0.027 \pm 0.002)$,

is in good agreement with the linear regression obtained by Gustafsson et al. (1999) using the abundances of F and G dwarfs by Edvardsson et al. (1993), who found

$[\mathrm{O} / \mathrm{Fe}]=(-0.36 \pm 0.02) \times[\mathrm{Fe} / \mathrm{H}]-(0.044 \pm 0.010)$,

and plausibly reflects that iron enrichment by $\mathrm{SNe} \mathrm{Ia}$ takes place in larger timescales when compared with the rapid production of oxygen by $\mathrm{SNe}$ II (as first suggested by Matteucci \& Greggio 1986).

5. The thin disk sequence at $[\mathrm{Fe} / \mathrm{H}]=0$ is slightly below zero. At $[\mathrm{Fe} / \mathrm{H}]=0$, we found a value of $[\mathrm{O} / \mathrm{Fe}]=$ $-0.027 \pm 0.002$, which is, in absolute value, slightly larger than the value we derived for the Sun (see Section 3.2). Similar results were found by Meléndez et al. (2009; an average $\langle[\mathrm{O} / \mathrm{Fe}]\rangle=-0.033 \pm 0.011$ for 11 stars $)$ and Ramírez et al. $(2009 ;\langle[\mathrm{O} / \mathrm{Fe}]\rangle=-0.015 \pm$ 0.006 for 22 stars), who used samples of solar twins. Similar studies based on F-, G-, and K-type stars in the solar neighborhood have also been conducted with compatible results, e.g., Gustafsson et al. (1999; $\langle[\mathrm{O} / \mathrm{Fe}]\rangle=-0.044 \pm 0.010$ for 57 stars), Ramírez et al. $(2013 ;\langle[\mathrm{O} / \mathrm{Fe}]\rangle=-0.02 \pm 0.04$ for 47 stars $)$, and Nissen et al. $(2014 ;\langle[\mathrm{O} / \mathrm{Fe}]\rangle=-0.016 \pm 0.04$ for 11 stars). A possible explanation for the observed offset can be found in Nieva \& Przybilla (2012), who suggested that the Sun was born at a distance around 5-6 kpc from the Galactic center, where higher metallicity values were reached earlier in the cosmic history of the Galaxy, and it 

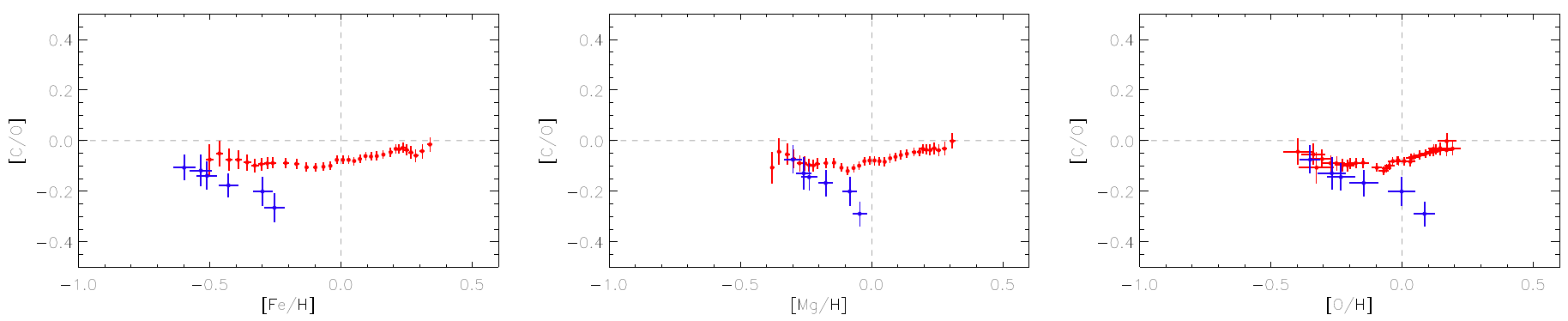

Figure 5. The $[\mathrm{C} / \mathrm{O}]$ vs. $[\mathrm{Fe} / \mathrm{H}]$ (left panel), $[\mathrm{Mg} / \mathrm{H}]$ (middle panel), and $[\mathrm{O} / \mathrm{H}]$ (right panel) diagrams for the thin (red) and thick (blue) disk samples. Oxygen abundances are from this paper, carbon abundances are from FR20, and $[\mathrm{Fe} / \mathrm{H}]$ and $[\mathrm{Mg} / \mathrm{H}]$ are from the GES iDR5.

has migrated outward during its lifetime of $4.5 \mathrm{Gyr}$ at the current position. An alternative explanation for this peculiarity is related to the formation of planetary systems like the solar one and, in particular, terrestrial planets (Meléndez et al. 2009).

As far as the $[\mathrm{O} / \mathrm{Mg}]-[\mathrm{Mg} / \mathrm{H}]$ trend is concerned (right panel of Figure 4), we can make the following considerations.

1. The decrease of $[\mathrm{O} / \mathrm{Mg}]$ with $[\mathrm{Mg} / \mathrm{H}]>0.0$ for the thin disk stars gives evidence of a different behavior of oxygen and magnesium at high metallicities, i.e., they do not evolve in lockstep, and indicates that, while oxygen is only enriched by CCSNe, magnesium might also be released, to some extent, by SNe Ia and/or AGB stars (Magrini et al. 2017; Ventura et al. 2018).

2. Both the thick and thin disk stars at $[\mathrm{Mg} / \mathrm{H}] \lesssim-0.1$ show trends almost flat with $[\mathrm{Mg} / \mathrm{H}]$ at $[\mathrm{O} / \mathrm{Mg}] \simeq 0$. These flat $[\mathrm{O} / \mathrm{Mg}]$ trends are in agreement with the results by Bensby et al. (2004; see their Figure 12), even if their thick disk values at $[\mathrm{Mg} / \mathrm{H}] \lesssim 0.1$ are almost leveled out at a level slightly higher than their thin disk data. The increase in $[\mathrm{O} / \mathrm{Mg}]$ for the thick disk stars with $[\mathrm{Mg} / \mathrm{H}] \gtrsim-0.1$ may be due to the poor statistic, a selection effect, or the presence of an $[\mathrm{O} / \mathrm{Mg}]$ gradient with the star distance from the Galactic plane, as also suggested by the results presented below. In fact, since the two bins with higher $[\mathrm{Mg} / \mathrm{H}]$ contain mainly stars at low $\left|Z_{\max }\right|$, their high $[\mathrm{O} / \mathrm{Mg}]$ values may be simply due to the negative vertical gradient of this abundance ratio with $\left|Z_{\max }\right|$ shown in the third panel in the right column of Figure 6. Therefore, at present, it is premature to draw any conclusions.

\subsection{The $[\mathrm{C} / \mathrm{O}]$ Trends with $[\mathrm{Fe} / \mathrm{H}],[\mathrm{Mg} / \mathrm{H}]$, and $[\mathrm{O} / \mathrm{H}]$}

The $[\mathrm{C} / \mathrm{O}]$ ratio in the Galactic disks has also been under debate and is highly relevant in a number of astrophysical scenarios, for example, to understand planet formation processes and the possible existence of terrestrial planets (e.g., Delgado Mena et al. 2010; Nissen 2013; Nissen et al. 2014; Brewer et al. 2017; Amarsi et al. 2019). In the context of this paper, it is particularly useful when discussing the origin and evolution of carbon in the Galaxy. Since oxygen is exclusively produced in massive stars on a relatively short timescale $\left(\sim 10^{7} \mathrm{yr}\right)$, the change in $[\mathrm{C} / \mathrm{O}]$ as a function of $[\mathrm{O} / \mathrm{H}]$ depends on the yields and timescales of carbon synthesized in different types of stars (Chiappini et al. 2003; Akerman et al. 2004; Carigi et al. 2005; Cescutti et al. 2009; Romano et al. 2020).
Figure 5 shows how $[\mathrm{C} / \mathrm{O}]$ changes as a function of $[\mathrm{Fe} / \mathrm{H}]$, $[\mathrm{Mg} / \mathrm{H}]$, and $[\mathrm{O} / \mathrm{H}]$ for the thin and thick disk stars. In particular, from Figure 5, we can observe the following.

1. There is a slight increment of $[\mathrm{C} / \mathrm{O}]$ with $[\mathrm{Fe} / \mathrm{H}],[\mathrm{Mg} /$ $\mathrm{H}]$, or $[\mathrm{O} / \mathrm{H}]$ for thin disk stars for $[\mathrm{Fe} / \mathrm{H}] \gtrsim-0.1$. Such an increase can be explained by taking into account a $\mathrm{C}$ production contribution from low-mass and/or massive stars at high metallicities due to their enhanced mass loss.

2. Thick disk stars show a decrease of $[\mathrm{C} / \mathrm{O}]$ with $[\mathrm{Fe} / \mathrm{H}]$, $[\mathrm{Mg} / \mathrm{H}]$, and $[\mathrm{O} / \mathrm{H}]$. A qualitatively similar behavior can be observed in the $[\mathrm{C} / \mathrm{O}]$ versus $[\mathrm{O} / \mathrm{H}]$ correlation of Stonkute et al. (2020, see their Figure 11), even if they interpreted it as due to the presence of a shift $(\sim-0.3$ dex) of their thick disk star sequence with respect to that for the thin disk. On the other hand, Bensby \& Feltzing (2006) found a flat behavior for the thick disk stars until $[\mathrm{O} / \mathrm{H}] \simeq 0$ and then an increase. In any case, we should point out that the thick disk star bin behavior could be an artifact in our case. Therefore, it would be desirable to expand the thick disk sample, aiming at verifying such a $[\mathrm{C} / \mathrm{O}]$ decrease and elucidating the effects of the steep vertical gradient of $[\mathrm{O} / \mathrm{Fe}]$ (see Figure 6 ) on the observed tendency.

\section{3. $[\mathrm{O} / \mathrm{H}],[\mathrm{O} / \mathrm{Fe}],[\mathrm{O} / \mathrm{Mg}]$, and $[\mathrm{C} / \mathrm{O}]$ Trends with $\mathrm{R}_{\text {med }}$ and $\left|Z_{\max }\right|$}

In this section, we present the trends of oxygen abundance ratios with the mean galactocentric distance, $R_{\text {med }}=0.5 \times$ $\left(R_{\min }+R_{\max }\right)$, and the maximum absolute distance from the Galactic plane, $\left|Z_{\max }\right|$, for the 333 thin disk and 18 thick disk stars for which stellar orbits were calculated in FR20.

Both observed radial and vertical stellar abundance distributions are particularly interesting tools to study the chemical enrichment history of the Galactic disks and their formation processes. Actually, the radial and vertical thin and thick disk star trends collect a lot of very important information to disentangle the embedded aspects of inflow, star formation, outflow, and radial and vertical mixing that gave rise to the present state of the Milky Way disk (for example, a radial gradient of the disk that is produced in an inside-out formation scenario can be partially reduced/canceled by a radial mixing process). The radial metallicity gradients of the Milky Way disk have been measured using a number of different stellar (e.g., Cepheids, open clusters) and interstellar medium tracers (H II regions, etc.) that represent the composition of the interstellar gas at the time they were formed (e.g., Cheng et al. 2012; Mikolaitis et al. 2014, and references therein) or the present time, respectively, thus probing the metallicity gradient 

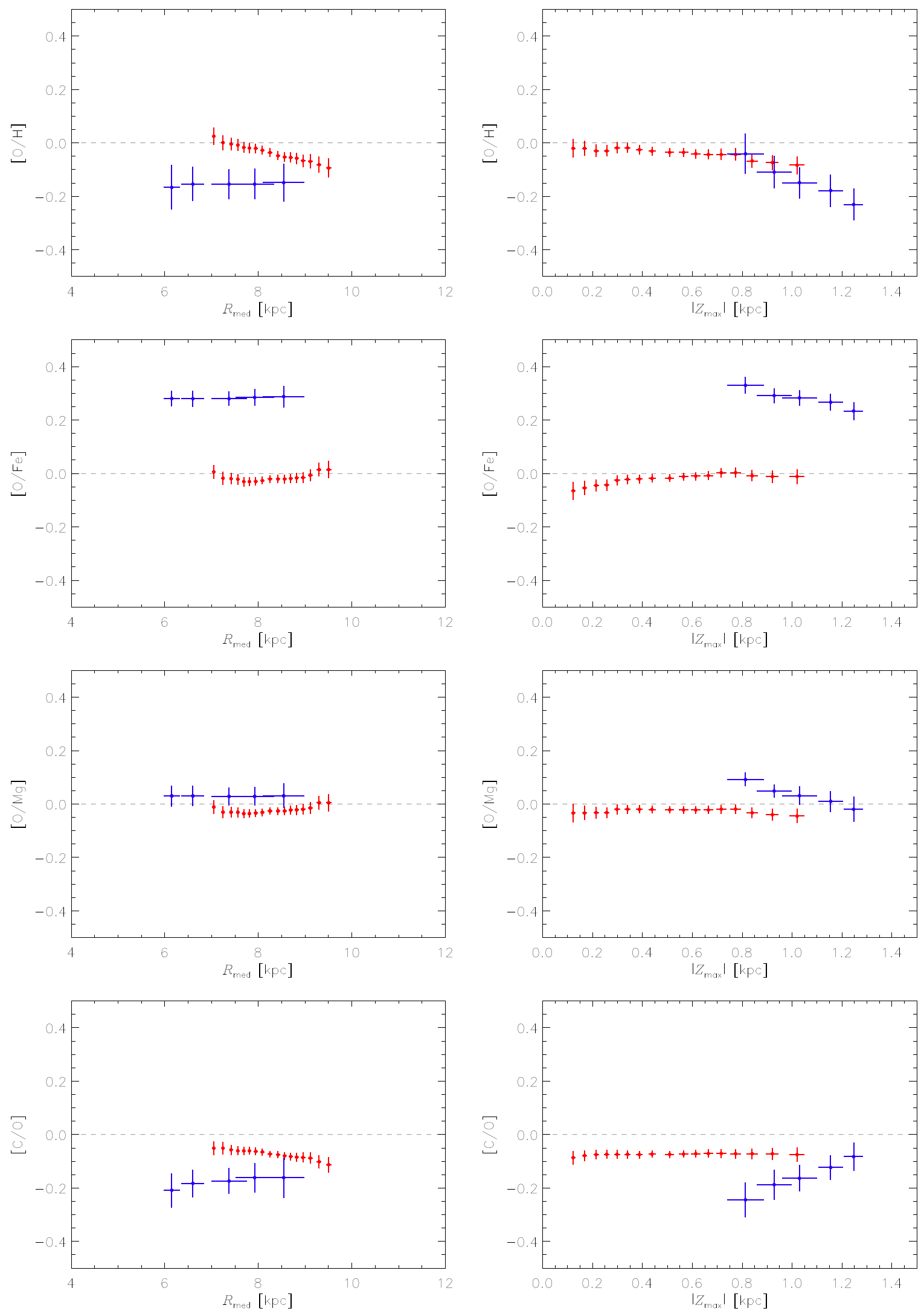

Figure 6. The $[\mathrm{O} / \mathrm{H}],[\mathrm{O} / \mathrm{Fe}],[\mathrm{O} / \mathrm{Mg}]$, and $[\mathrm{C} / \mathrm{O}]$ (top to bottom) vs. $R_{\text {med }}$ (left panels) and $\left|Z_{\max }\right|$ (right panels) diagrams for thin (red) and thick (blue) disk star samples.

at different epochs. In addition, the vertical gradients of the global metallicity and different elements have also been derived by using different input databases (see, for example,
Boeche et al. 2014; Schlesinger et al. 2014; Magrini et al. 2017, and references therein). One of the causes of the different gradient values, both radial and vertical, reported in the 


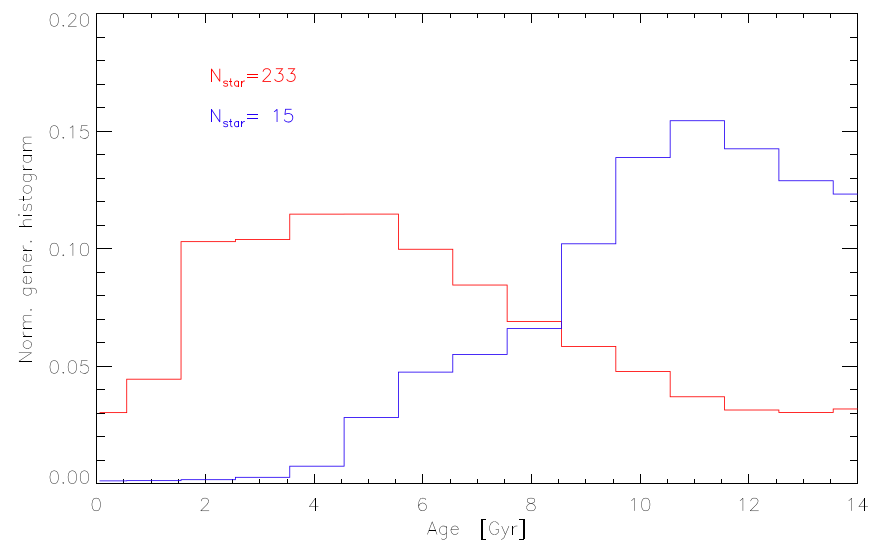

Figure 7. Normalized generalized age histograms for thin (red) and thick (blue) disk star samples.

literature might be attributed to different input data. In fact, gradients may be affected by the chemical evolution of the Galaxy (i.e., by the time the analyzed objects were formed), local inhomogeneities, moving groups, and stellar streams. Moreover, the measured gradients may be biased by systematics and/or inhomogeneities in the spatial and age distributions of the input data samples; i.e., derived vertical gradients may be more affected by stars located in the outer or inner galactic radius, $R$, and/or younger or older stars if the $Z$ bins do not contain evenly distributed objects in $R$ and age. Therefore, the interpretation of the derived gradients as result of the star formation history of the galactic disk(s) is very challenging. In the following, we will take advantage of the fact that the stars in our thin and thick disk stellar samples display, to some extent, different dependencies on $R_{\text {med }}$ and $\left|Z_{\text {max }}\right|$ and span different age ranges (see Figures 6 and 7), but it is worthwhile to remark that our interpretation of the results may still be affected by systematics, particularly for the thick disk stellar sample.

In Figure 6, we show the radial and vertical gradients of [O/ $\mathrm{H}],[\mathrm{O} / \mathrm{Fe}],[\mathrm{O} / \mathrm{Mg}]$, and $[\mathrm{C} / \mathrm{O}]$ for the thin and thick disk star samples, again using running average bins as in Figures 4 and 5 .

As far as the trends as a function of $R_{\text {med }}$ are concerned, we recall that FR20 did not have deviations from axisymmetry in their Galactic potential like those introduced by the bar and the spiral arms and did not take into account radial stellar migration. However, $R_{\text {med }}$ values can provide an indication of the stellar birthplaces (Edvardsson et al. 1993; Rocha-Pinto et al. 2004), which could be used, at least, in a differential form.

As can be seen, the thin and thick disk stars fall in different $\left|Z_{\text {max }}\right|$ ranges: most of the thin disk bins have $\left|Z_{\text {max }}\right|<0.8 \mathrm{kpc}$, while all of the thick disk bins have $\left|Z_{\max }\right|>0.8 \mathrm{kpc}$. Moreover, as discussed in the next section, most of the thin disk star bins have ages $<10 \mathrm{Gyr}$, while all of the thick disk objects have ages $>10$ Gyr (see Figure 8 ). Therefore, the trends with $R_{\text {med }}$ of our thin and thick disk stars may give hints of the radial gradients of relatively young stars at low $\left|Z_{\text {max }}\right|$ and old stars at higher $\left|Z_{\max }\right|$, respectively.

By inspection of Figure 6, we can observe the following.

1. The $[\mathrm{O} / \mathrm{H}]$ and $[\mathrm{C} / \mathrm{O}]$ show negative gradients as a function of $R_{\text {med }}$ for the thin disk sample. The abundance ratios $[\mathrm{O} / \mathrm{H}]$ and $[\mathrm{C} / \mathrm{O}]$ decrease significantly with the mean galactocentric distance, while the thick disk sample exhibits flat trends or perhaps very mild positive gradients, as indicated by the fits of Table 3 . The positive radial gradient of $[\mathrm{C} / \mathrm{O}]$ for the thick disk stars might not be real, since it could actually be a result of the fact that our data bins correspond to groups of stars with different average $\left|Z_{\max }\right|$ values and are actually reflecting the presence of the steep $[\mathrm{C} / \mathrm{O}]$ vertical gradient shown in the bottom right panel of Figure 6. A dichotomy between thin and thick disk stars was found by several authors (e.g., Allende Prieto et al. 2006; Katz et al. 2011; Cheng et al. 2012; Boeche et al. 2013; Anders et al. 2014; Mikolaitis et al. 2014; RecioBlanco et al. 2014; Hayden et al. 2015, 2018; Kordopatis et al. 2015) and could indicate that either the thick disk formed from a well-mixed interstellar material or, if gradients were present, they disappeared because of a migration effect more efficient in the thick disk than in the thin one (Minchev et al. 2013). Esteban et al. (2005), using recombination lines from echelle spectrophotometry of eight $\mathrm{H}$ II regions with galactocentric distances in the range $6-10 \mathrm{kpc}$, derived a value of $-0.044 \pm 0.010 \mathrm{dex} \mathrm{kpc}^{-1}$ for the $[\mathrm{O} / \mathrm{H}]$ radial gradient. Rudolph et al. (2006), also based on the analysis of $\mathrm{H}$ II regions, derived values of -0.060 and $-0.041 \mathrm{dex} \mathrm{kpc}^{-1}$ by using optical and infrared data, respectively. More recently, Wang (2019) used spectra of $101 \mathrm{H}$ II regions in the Galactic anticenter area from the Large Sky Area Multi-Object Fiber Spectroscopic Telescope (LAMOST) and derived the oxygen abundance gradient with a slope of $-0.036 \pm 0.004 \mathrm{dex} \mathrm{kpc}^{-1}$. Wenger et al. (2019), using the National Radio Astronomy Observatory Karl G. Jansky Very Large Array to observe $\sim 8-10 \mathrm{GHz}$ hydrogen radio recombination line and radio continuum emission toward 82 Galactic H II regions, found an oxygen abundance gradient across the Milky Way disk with a slope of $-0.052 \pm 0.004 \mathrm{dex} \mathrm{kpc}^{-1}$. All of these values are in good agreement, within the uncertainties, with our slope value (the small errors given in Table 3 are due to the fact that we fitted the abundances of the average bins and not the individual stars). Of course, we compare our gradients with those from $\mathrm{H}$ II regions, which actually reflect the present situation, while in our trends, we mix stars of somewhat different ages with only a rough separation between young/thin and old/thick disk populations. As a further comparison, Cescutti et al. (2007) found a gradient of $-0.035 \mathrm{dex} \mathrm{kpc}^{-1}$ for $\mathrm{O}$ for a galactocentric distance of 4-14 kpc, in good agreement with our results.

A negative radial gradient of $[\mathrm{C} / \mathrm{O}]$ was observed by Esteban et al. (2005) from $\mathrm{H}$ II region recombination lines. They found a steeper gradient, $\frac{d[\mathrm{C} / \mathrm{O}]}{d \mid Z_{\max } !}=-0.058 \pm$ 0.018 , than us. On the theoretical side, Carigi et al. (2005) showed that, by varying carbon, nitrogen, and oxygen yields, Galactic chemical evolution models predict slopes between -0.005 and $-0.068 \mathrm{dex} \mathrm{kpc}^{-1}$.

We notice that most of the $[\mathrm{O} / \mathrm{H}]$ and all of the $[\mathrm{C} / \mathrm{O}]$ values of the thin disk bins are negative, suggesting that solar neighborhood stars have less oxygen and $\mathrm{C} / \mathrm{O}$ than the Sun. To explain these results, Nieva \& Przybilla (2012) stated that the Sun may be an immigrant to its current Galactic neighborhood; i.e., the birthplace of the Sun could be at an inner galactocentric distance $(R=\sim 5-6 \mathrm{kpc})$ where higher metallicity values were reached earlier in cosmic history. If this is true, they claimed that "a telltaling signature is left only in the $\mathrm{C} / \mathrm{O}$ ratio" (but they did not discuss $[\mathrm{O} / \mathrm{H}])$. Our results suggest that a solar $[\mathrm{O} / \mathrm{H}]$ value 

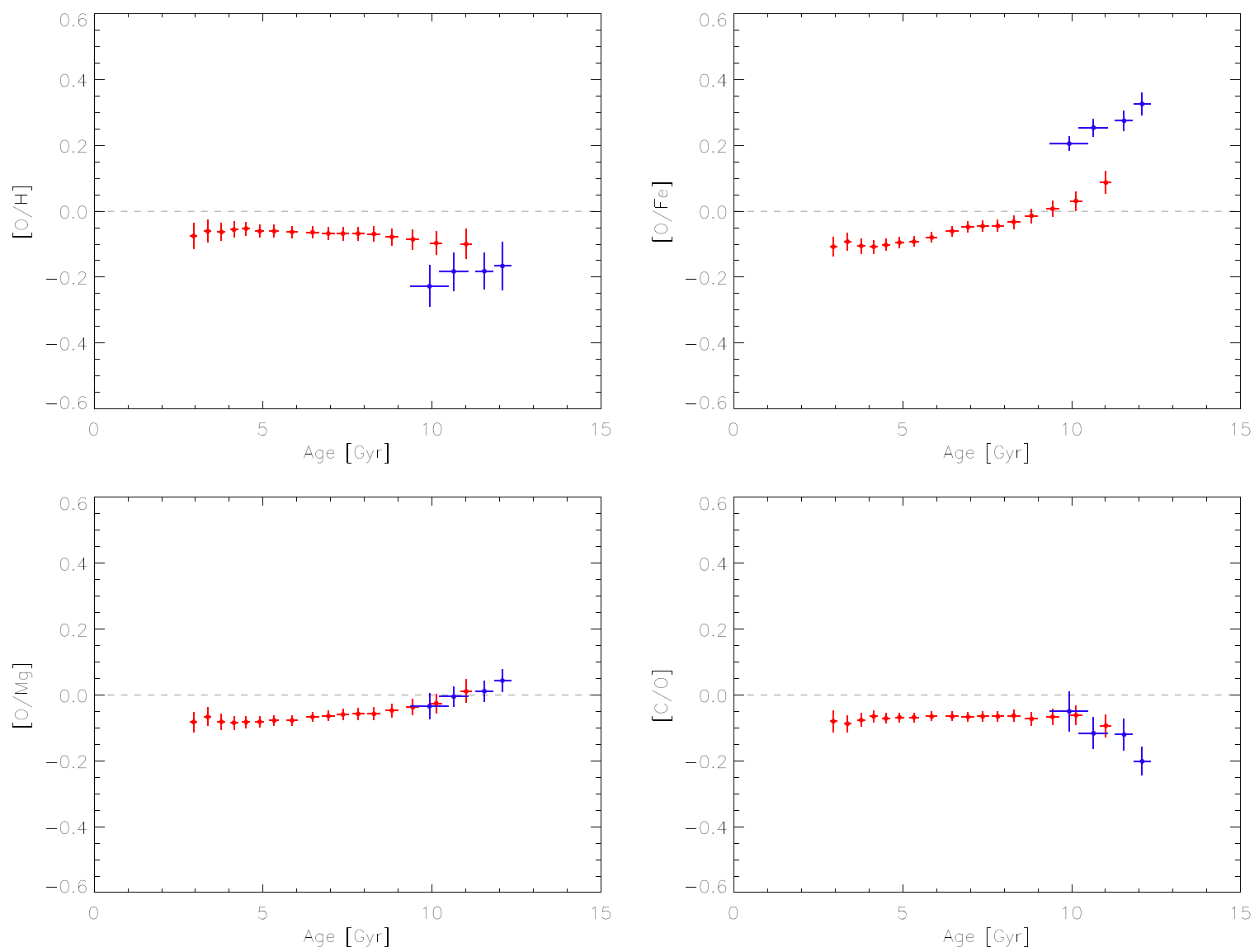

Figure 8. The $[\mathrm{O} / \mathrm{H}]$ (top left panel), $[\mathrm{O} / \mathrm{Fe}]$ (top right panel), $[\mathrm{O} / \mathrm{Mg}]$ (bottom left panel), and $[\mathrm{C} / \mathrm{O}]$ (bottom right panel) vs. age diagrams for thin (red) and thick (blue) disk samples.

should be characteristic of $R_{\text {med }}=\sim 7 \mathrm{kpc}$, while solar [C/ O] would imply $R_{\text {med }} \lesssim 6 \mathrm{kpc}$, but we recall again that, due to the simplified Galactic potential used by FR20 to compute stellar orbits, $R_{\text {med }}$ gives only a rough indication of the actual stellar birthplaces.

2. The $[\mathrm{O} / \mathrm{Fe}]$ and $[\mathrm{O} / \mathrm{Mg}]$ versus $R_{\text {med }}$ show flat (or slightly positive for the thin disk star bins) trends (see Table 3). Mikolaitis et al. (2014) found a slightly positive slope of $[\alpha /$ $\mathrm{M}]$ in their clean thin disk sample $\left(0.014 \pm 0.004 \mathrm{dex} \mathrm{kpc}^{-1}\right)$ and a flat trend in their clean thick disk sample $(-0.005 \pm$ $0.006 \mathrm{dex} \mathrm{kpc}^{-1}$ ). Unfortunately, their results cannot be directly compared with our $[\mathrm{O} / \mathrm{Fe}]$ trends, since their $\alpha$ elements do not include oxygen. The $[\mathrm{O} / \mathrm{Mg}]$ versus $R_{\text {med }}$ shows even flatter trends and a much smaller systematic difference between the values for thick and thin disk stars than the $[\mathrm{O} / \mathrm{Fe}]$. These results might indicate that $\mathrm{SNe}$ Ia not only produce an enrichment of $\mathrm{Fe}$ in the thin disk but also a small increase of $\mathrm{Mg}$, while oxygen seems to be enriched only by CCSNe.

For the abundance trends as a function of $\left|Z_{\max }\right|$, we identify the following features.

1. The $[\mathrm{O} / \mathrm{H}]$ bins show a negative vertical gradient with slope increasing from lower (thin disk star bins) to higher (thick disk star bins) $\left|Z_{\max }\right|$ (see Table 4, where the thin disk fits refer to bins with $\left|Z_{\max }\right|<0.8 \mathrm{kpc}$ ). Similar behaviors for $\frac{d[\mathrm{Fe} / \mathrm{H}]}{d\left|Z_{\max }\right|}$ and $\frac{d[\mathrm{Si} / \mathrm{H}]}{d\left|Z_{\max }\right|}$ were found by Boeche et al. (2014). In an analysis of $\alpha$-elements, Mikolaitis et al. (2014) found $\frac{d \alpha}{d|Z|}=+0.036 \pm 0.006 \mathrm{dex} \mathrm{kpc}^{-1}$ for their thin disk clean sample. However, we want to remark that these authors did not include oxygen in their $\alpha$ mixture.

2. The $[\mathrm{O} / \mathrm{Fe}]$ and $[\mathrm{O} / \mathrm{Mg}]$ thin disk star bins show positive trends with $\left|Z_{\max }\right|$ (see Table 4 ) that could be explained by the increasing contribution of iron and magnesium by $\mathrm{SNe}$ Ia (we recall that our stars at low $\left|Z_{\max }\right|$ are also the youngest ones, as shown in Figure 14 of FR20). The thick disk star bins have negative trends like $[\mathrm{O} / \mathrm{H}]$ but with lower slopes. The fact that $[\mathrm{O} / \mathrm{Mg}]$ shows a smaller difference between the average values of thick and thin disk star bins than $[\mathrm{O} / \mathrm{Fe}]$ (as already seen in the radial gradient panels) is a consequence of the greater yields of iron than magnesium in SNe Ia.

3. The $[\mathrm{C} / \mathrm{O}]$ shows an almost flat trend for the thin disk star bins, suggesting that both carbon and oxygen were produced by massive stars. The positive trend of the thick disk star bins reflects the steep negative trend of $[\mathrm{O} / \mathrm{H}]$ with $\left|Z_{\max }\right|$ shown in the top right panel of Figure $6 .{ }^{24}$

\section{4. $[\mathrm{O} / \mathrm{H}],[\mathrm{O} / \mathrm{Fe}],[\mathrm{O} / \mathrm{Mg}]$, and $[\mathrm{C} / \mathrm{O}]$ Trends with Age}

Nominal ages for 233 and 15 stars belonging to our thin and thick disk star samples, respectively, were given by FR20. The thick disk stars are, in general, older than members of the thin

\footnotetext{
${ }^{24}$ The flat behavior of $[\mathrm{C} / \mathrm{H}]$ versus $\left|Z_{\max }\right|$ for the same objects, as checked by the authors, does not affect the $[\mathrm{C} / \mathrm{O}]$ trend.
} 
Table 3

Radial Gradients

\begin{tabular}{|c|c|c|c|c|c|c|c|c|}
\hline & $\begin{array}{c}\frac{d[\mathrm{O} / \mathrm{H}]}{d R_{\text {med }}} \\
\left(\mathrm{dex} \mathrm{kpc}^{-1}\right)\end{array}$ & $\chi_{\mathrm{r}}$ & $\begin{array}{c}\frac{d[\mathrm{O} / \mathrm{Fe}]}{d R_{\mathrm{med}}} \\
\left(\mathrm{dex} \mathrm{kpc}^{-1}\right)\end{array}$ & $\chi_{\mathrm{r}}$ & $\begin{array}{c}\frac{d[\mathrm{O} / \mathrm{Mg}]}{d R_{\mathrm{med}}} \\
\left(\mathrm{dex} \mathrm{kpc}^{-1}\right)\end{array}$ & $\chi_{\mathrm{r}}$ & $\begin{array}{c}\frac{d[\mathrm{C} / \mathrm{O}]}{d R_{\text {med }}} \\
\left(\operatorname{dex~kpc}^{-1}\right)\end{array}$ & $\chi_{\mathrm{r}}$ \\
\hline Thin & $-0.041 \pm 0.001$ & 0.145 & $0.014 \pm 0.004$ & 0.269 & $0.009 \pm 0.002$ & 0.193 & $-0.020 \pm 0.001$ & 0.146 \\
\hline
\end{tabular}

Table 4

Vertical Gradients

\begin{tabular}{|c|c|c|c|c|c|c|c|c|}
\hline & $\begin{array}{c}\frac{d[\mathrm{O} / \mathrm{H}]}{d\left|Z_{\max }\right|} \\
\left(\mathrm{dex} \mathrm{kpc}^{-1}\right)\end{array}$ & $\chi_{\mathrm{r}}$ & $\begin{array}{c}\frac{d[\mathrm{O} / \mathrm{Fe}]}{d\left|Z_{\max }\right|} \\
\left(\mathrm{dex} \mathrm{kpc}^{-1}\right)\end{array}$ & $\chi_{\mathrm{r}}$ & $\begin{array}{c}\frac{d[\mathrm{O} / \mathrm{Mg}]}{d\left|Z_{\max }\right|} \\
\left(\mathrm{dex} \mathrm{kpc}^{-1}\right)\end{array}$ & $\chi_{\mathrm{r}}$ & $\begin{array}{c}\frac{d[\mathrm{C} / \mathrm{O}]}{d\left|Z_{\max }\right|} \\
\left(\operatorname{dex~kpc}^{-1}\right)\end{array}$ & $\chi_{\mathrm{r}}$ \\
\hline Thin & $-0.04 \pm 0.01$ & 0.229 & $0.09 \pm 0.01$ & 0.350 & $0.020 \pm 0.004$ & 0.234 & $0.007 \pm 0.001$ & 0.053 \\
\hline
\end{tabular}

disk. Figure 7 shows the normalized, generalized age histograms of both populations, built by summing the individual age probability distributions, for the thin (red) and thick (blue) disk stars. The extended wings and, therefore, the overlap of the two distributions are likely due to the large uncertainties affecting individual stellar ages. Most of the thin disk stars span a range from 2 to $8 \mathrm{Gyr}$, while the thick disk stars are mostly older than 8 Gyr. This is in agreement with the common understanding of Galactic chemical evolution based on serial and two-infall models (e.g., Chiappini et al. 1997; Calura \& Menci 2009; Romano et al. 2010; Grisoni et al. 2017) and chemical abundance studies matched with asteroseismologic age determinations, which suggest a delay of $\sim 4$ Gyr between the first and second accretion episodes that were the origin of the Milky Way disks (Haywood et al. 2015; Snaith et al. 2015; Spitoni et al. 2019).

Figure 8 shows the trends of $[\mathrm{O} / \mathrm{H}],[\mathrm{O} / \mathrm{Fe}],[\mathrm{O} / \mathrm{Mg}]$, and $[\mathrm{C} / \mathrm{O}]$ versus age. As for previous figures, we plot in red and blue the thin and thick disk stellar samples, with the bins constructed using a running average.

In Figure 8, we identify the following properties of the abundance ratios versus age.

1. A difference in the age intervals spanned by thin (in general younger) and thick (older) disk star bins is clearly evident with only a small overlap between 10 and 11 Gyr. Recalling the intrinsic uncertainties in FR20 age estimates, it is possible that such an overlap could actually be less pronounced.

2. Both thin and thick disk stars show almost flat trends of $[\mathrm{O} / \mathrm{H}]$ versus age. A similar behavior was observed by Delgado Mena et al. (2019), who, by using the oxygen abundances from Bertran de Lis et al. (2015), derived two similar flat trends of $[\mathrm{O} / \mathrm{H}]$ with age for thin and thick disk stars for the HARPS-GTO sample. The small negative slope $(-0.004 \pm 0.001)$ for our thin disk star bins and the slightly larger positive one $(0.028 \pm 0.004)$ for our thick disk star bins with age are likely to be remnants of the radial and vertical gradients (plus selection effects) shown in Figure 6 (red points in the top left panel and blue points in the top right panel, respectively). The higher average value for the thin disk stars indicates that the thin disk formation started only after many CCSNe had already produced $\mathrm{O}$ enrichment of the interstellar medium.

3. Both thin and thick disk stars show increasing trends of $[\mathrm{O} / \mathrm{Fe}]$ and $[\mathrm{O} / \mathrm{Mg}]$ with age with an almost zero slope for the youngest objects. Since our stars do not show a strong spatial gradient in $[\mathrm{O} / \mathrm{Fe}]$ and $[\mathrm{O} / \mathrm{Mg}]$ (see the middle panels of Figure 6), we think that they really indicate abundance ratio trends with age. There is a jump from higher to lower $[\mathrm{O} / \mathrm{Fe}]$ values in the common age region, while the $[\mathrm{O} / \mathrm{Mg}]$ sequences merge smoothly. The steeper trends at the oldest ages confirm that oxygen is indeed mainly produced in the early phases of Galaxy formation by massive stars. Such correlations were also in other $[\alpha / \mathrm{Fe}]$ ratios (see, for example, Nissen \& Gustafsson 2018; Delgado Mena et al. 2019, and references therein). In fact, steep age trends at old ages and flat trends at young ages should be expected for [O/ $\mathrm{Fe}]$ if the CCSNe are the predominant (or even the only) suppliers of oxygen in the interstellar medium, while a less clear behavior can be foreseen in the case of other $\alpha$ elements, which are also partially released by $\mathrm{SNe}$ Ia (e.g., Carigi et al. 2005; Nomoto et al. 2013). We also found steeper slopes for $[\mathrm{O} / \mathrm{Fe}]$ than for $[\mathrm{O} / \mathrm{Mg}]$, which is consistent with the fact that $\mathrm{SNe}$ Ia release much more iron than magnesium in the interstellar medium. The slopes of $[\mathrm{O} / \mathrm{Fe}]$ and $[\mathrm{O} / \mathrm{Mg}]$ versus age for our thin disk star bins are 0.019 and 0.01 dex $\mathrm{Gyr}^{-1}$, respectively, and in qualitative agreement with the results by Delgado Mena et al. (2019), who found 0.026 and 0.02 dex $\mathrm{Gyr}^{-1}$ using a HARPS-GTO sample. The smooth merger of the thin and thick trends of $[\mathrm{O} / \mathrm{Mg}]$ seems to indicate that $[\mathrm{O} / \mathrm{Mg}]$ should be preferred to $[\mathrm{O} / \mathrm{Fe}]$ as a proxy of age, and its shape suggests that the correlation is nonlinear in the whole age interval from 3 to $12 \mathrm{Gyr}$.

4. The $[\mathrm{C} / \mathrm{O}]$ trends show a steep decrease for the oldest objects (age $\gtrsim 10 \mathrm{Gyr}$ ) and an almost flat trend for younger objects. As in the case of $[\mathrm{O} / \mathrm{H}]$, this behavior with age can actually be the result of hidden spatial $[\mathrm{C} /$ O] gradients (see the bottom panels of Figure 6). This interpretation agrees with the results by Nissen (2015), who, using HARPS spectra of 21 solar twins in the solar neighborhood, showed that the $\mathrm{C} / \mathrm{O}$ ratio evolves very little with time, although the $[\mathrm{C} / \mathrm{Fe}]$ and $[\mathrm{O} / \mathrm{Fe}]$ ratios evolve significantly. He showed a good agreement between the data (see his Figure 11) and the predictions of the Galactic chemical evolution model by Gaidos (2015), in which the dominant contribution of $\mathrm{C}$ and $\mathrm{O}$ comes from massive stars. It is also argued that $\mathrm{C} / \mathrm{O}$ has only a moderate rise during the first $\sim 5$ Gyr due to an 

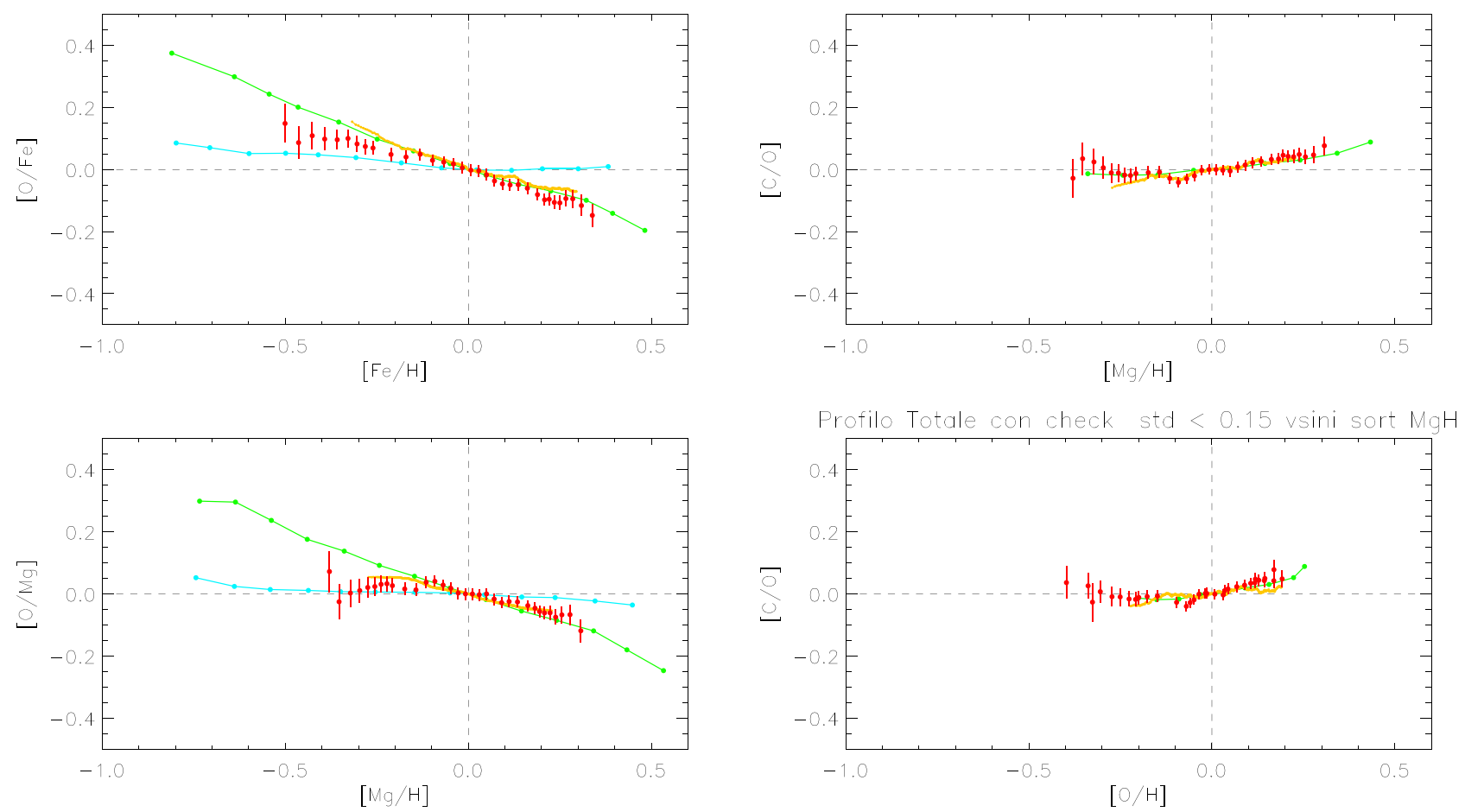

Figure 9. Comparison between our $[\mathrm{O} / \mathrm{Fe}]-[\mathrm{Fe} / \mathrm{H}]$ (top left panel), $[\mathrm{O} / \mathrm{Mg}]-[\mathrm{Mg} / \mathrm{H}]$ (bottom left panel), $[\mathrm{C} / \mathrm{O}]-[\mathrm{Mg} / \mathrm{H}]$ (top right panel), and $[\mathrm{C} / \mathrm{O}]-[\mathrm{O} / \mathrm{H}]$ (bottom right panel) trends and the WE19, GR19, and BR16 ones. Red symbols represent our thin disk star bins, as in previous figures. Solid lines represent trends of thin disk stars from WE19 (light blue), GR19 (green), and BR16 (yellow) data. In the two plots of [C/O] abundance ratios, WE19 data are not available.

increasing contribution of carbon from stars of lower mass. This is also in agreement with what was found by FR20.

\section{Comparison with Other Surveys}

In this section, we compare some of our results presented in Sections 4.1 and 4.2 with the median trends derived for other large stellar samples of the solar neighborhood, namely, APOGEE (Weinberg et al. 2019, hereafter WE19), GALAH DR2 (Griffith et al. 2019, hereafter GR19), and one constructed upon HIRES Keck data (Brewer \& Fischer 2016, hereafter BR16). WE19, who adopted Mg rather than Fe as their reference element, separated their stars into two populations (15 and 996 low-[Fe/Mg] or high- $\alpha$ and high- $[\mathrm{Fe} / \mathrm{Mg}]$ or low- $\alpha$, respectively, with $[\mathrm{O} / \mathrm{Mg}]$ determinations) on the basis of their $[\mathrm{Fe} / \mathrm{Mg}]$ and derived the median trends of $[\mathrm{X} / \mathrm{Mg}]$ versus $[\mathrm{Mg} / \mathrm{H}]$ in each population. Likewise, GR19 found 220 and 1146 high- and low- $\alpha$ stars, respectively (renamed by the authors low- and high-Ia stars), with trustworthy carbon and oxygen abundances and derived median sequences of $[\mathrm{X} / \mathrm{Mg}]$ versus $[\mathrm{Mg} / \mathrm{H}]$. We adopted the same kind of approach in order to separate the stars in BR16's sample. Actually, we used the chemical criterion described in FR20; i.e., in the same range of $T_{\text {eff }}$ and $\log g$ of our GES stars, we used the positions of the BR16 stars in the $[\mathrm{Mg} / \mathrm{Fe}]-[\mathrm{Fe} / \mathrm{H}]$ plane to obtain two samples of 460 and 22 low- and high- $\alpha$ stars, respectively. In the following, we will consider the WE19, GR19, and BR16 high- $\alpha$ (low-Ia) and low- $\alpha$ (high-Ia) stars as analogous to our thin and thick disk sample, respectively.

In Figure 9, we compare our $[\mathrm{O} / \mathrm{Fe}]-[\mathrm{Fe} / \mathrm{H}],[\mathrm{O} / \mathrm{Mg}]-[\mathrm{Mg} /$ $\mathrm{H}],[\mathrm{C} / \mathrm{O}]-[\mathrm{Mg} / \mathrm{H}]$, and $[\mathrm{C} / \mathrm{O}]-[\mathrm{O} / \mathrm{H}]$ trends with those from WE19, GR19, and BR19. Because of the small stellar sample of thick disk stars, we focus on the thin disk members.
As in previous figures, red points are used to identify our thin disk star bins, while solid lines represent the trends from WE19 (light blue), GR19 (green), and BR16 (yellow). Zero-point offsets were applied by GR19 to their GALAH DR2 data points and trends so that stars with $[\mathrm{Fe} / \mathrm{Mg}]=[\mathrm{Mg} / \mathrm{H}] \simeq 0$ also have $[\mathrm{X} / \mathrm{Mg}] \simeq 0$. To make the comparison easier, we adopted a similar approach and also computed the zero-point offsets for our data and those of WE19 and BR16 in order to have $[\mathrm{O} / \mathrm{Fe}]$ and $[\mathrm{O} / \mathrm{Mg}] \simeq 0$ for thin disk $($ low- $\alpha$ ) stars with $[\mathrm{Fe} / \mathrm{H}]$ or $[\mathrm{Mg} / \mathrm{H}] \simeq 0$. The data illustrated in Figure 9 are plotted after applying the proper zero offset.

From the left panels, we can clearly see that the WE19 trends of $[\mathrm{O} / \mathrm{Fe}]$ and $[\mathrm{O} / \mathrm{Mg}]$ differ significantly from the others. The WE19 $[\mathrm{O} / \mathrm{Fe}]$ versus $[\mathrm{Fe} / \mathrm{H}]$ and $[\mathrm{O} / \mathrm{Mg}]$ versus $[\mathrm{Mg} / \mathrm{H}]$ trends are almost flat, while the GR19 and BR16 trends are both sloped and nearly superimposed for $[\mathrm{Fe} / \mathrm{H}] \gtrsim-0.3$ and $[\mathrm{Mg} / \mathrm{H}] \gtrsim-0.1$ dex, respectively. Our data agree with the GR19 and BR16 results for $[\mathrm{Fe} / \mathrm{H}] \gtrsim-0.15$ and $[\mathrm{Mg} / \mathrm{H}] \gtrsim-0.1$ dex but fall between the GR19 and WE19 $[\mathrm{O} / \mathrm{Fe}]$ trends for lower $[\mathrm{Fe} / \mathrm{H}]$ and the BR16 and WE19 $[\mathrm{O} /$ $\mathrm{Mg}]$ trends for lower $[\mathrm{Mg} / \mathrm{H}]$, eventually merging with WE19 for $[\mathrm{Mg} / \mathrm{H}] \gtrsim-0.35$ dex.

In the right panels of Figure 9, we compare our [C/O] versus $[\mathrm{Mg} / \mathrm{H}]$ and $[\mathrm{O} / \mathrm{H}]$ thin disk star bins with the corresponding trends by GR19 and BR $16 .{ }^{25}$ In both diagrams, there is very good agreement of our data with the GR19 and BR16 trends, which also agree very well with each other.

Several things can explain the observed differences between the trends shown in Figure 9. For instance, different thresholds

\footnotetext{
25 APOGEE also measures C, but WE19 did not present carbon results, since their stars are mainly giants where original carbon surface abundances are altered by mixing with internal processed material during the first dredge-up phase (see Iben 1965); therefore, no WE19 trends are present in the right panels.
} 
in the $[\mathrm{Mg} / \mathrm{Fe}]-[\mathrm{Fe} / \mathrm{H}]$ plane adopted by GR19 and WE19 to separate the thin disk stars from the thick disk stellar bins with respect to those used by us may lead to slightly higher mean values of the $[\mathrm{Mg} / \mathrm{H}]$ ratio at a given $[\mathrm{Fe} / \mathrm{H}]$ value. Moreover, we recall that our two samples were selected not using a pure chemical criterion. Therefore, the already emphasized wellknown problem of the different definition of the Galactic thin and thick disk may have introduced inhomogeneity in the trends we are comparing. It is also important to bear in mind that stellar abundances depend in different ways on the galactic position and age of the stars. It was stated by WE19 that their $[\mathrm{X} / \mathrm{H}]$ trends with $[\mathrm{Mg} / \mathrm{H}]$ do not vary with the different Galactic positions; it cannot be excluded that the differences in the WE19, GR19 and BR16 trends and in our data can be due to the different mixture of radial and vertical positions and ages in the studied samples.

\section{Summary and Conclusions}

We have measured oxygen abundances of 516 FGK dwarf stars in the Galactic disk(s). The stellar sample was extracted from the GES iDR5 and constitutes a subsample of the FGK dwarf set whose carbon abundances were derived in FR20. In this paper, we derived the oxygen abundances by using the spectral synthesis of the forbidden oxygen line at $6300 \AA$, taking special care to account for the contribution to the observed blend of the two isotopic lines of Ni I at $6300.336 \AA$. Only those stars with spectra unaffected by telluric lines and/or bad removal of the sky emission line in the spectral region were considered. The abundance determinations ( $[\mathrm{O} / \mathrm{Fe}]$ ) were conducted through a comparison of the observed spectra with "on-the-fly" synthetic spectra computed with the SPECTRUM code from fully consistent atmosphere structures obtained by using the ATLAS12 program.

In order to discriminate between the thin and thick disk objects, we used three different selection approaches based on (a) the stellar positions in the $[\mathrm{Mg} / \mathrm{Fe}]-[\mathrm{Fe} / \mathrm{H}]$ plane, (b) the stellar Galactic velocities, and (c) orbital parameters. Then, we merged the results of these three methodologies, and, after discarding the few stars with discordant classifications, we obtained two samples of 376 and 20 stars classified, by at least one of the three methods, as thin and thick disk stars, respectively. In this paper, we compared the stars in the two samples by deriving trends of $[\mathrm{O} / \mathrm{H}],[\mathrm{O} / \mathrm{Fe}],[\mathrm{O} / \mathrm{Mg}]$, and $[\mathrm{C} /$ $\mathrm{O}]$ versus $[\mathrm{Fe} / \mathrm{H}]$ and $[\mathrm{Mg} / \mathrm{H}]$ and, for the stars with Galactic orbits and ages computed in FR20, also versus the stellar position in and above the Galactic plane and age. Our main results can be summarized as follows.

- Our thin and thick disk stars span different age intervals, with the thick disk members being older than those of the thin disk, as already found in many previous studies.

- The $[\mathrm{O} / \mathrm{H}]$ values versus $[\mathrm{Fe} / \mathrm{H}]$ show a large scatter with a reduced $\left.\sqrt{(} \chi^{2}\right)$ of the linear fits of $\sim 3$, suggesting that a significant part of the observed scatter may be astrophysical.

- Similar to other $\alpha$-elements, oxygen abundance ratios ([O/ $\mathrm{H}],[\mathrm{O} / \mathrm{Fe}]$ versus $[\mathrm{Fe} / \mathrm{H}]$ ) show systematic differences between thin and thick disk stars: (i) thick disk stars have a larger $\mathrm{O}$ abundance than thin disk stars at the same $[\mathrm{Fe} / \mathrm{H}]$ in the range covered by our data, and (ii) thin disk stars show a clear monotonic decreasing trend of $[\mathrm{O} / \mathrm{Fe}]$ with increasing metallicity, even at the metal-rich regime, in agreement with previous results (e.g., Castro et al. 1997; Chen et al. 2003; Bensby et al. 2004, 2014; Ecuvillon et al. 2006; Amarsi et al. 2019) and Galactic chemical evolution models (e.g., Chiappini et al. 2003). This last result suggests that the oxygen enrichment of the interstellar medium is produced only by CCSNe, with no evidence of contributions from $\mathrm{SNe}$ Ia or AGB stars that would cause a flattening of $[\mathrm{O} / \mathrm{Fe}]$ at $[\mathrm{Fe} / \mathrm{H}] \simeq 0$ like that observed in other $\alpha$-elements (Bensby et al. 2004, 2014).

- At $[\mathrm{Mg} / \mathrm{H}]>0.0$, the thin disk stars show a decrease of $[\mathrm{O} / \mathrm{Mg}]$ with $[\mathrm{Mg} / \mathrm{H}]$, indicating that oxygen and magnesium do not evolve in lockstep. Hence, these chemical species should have different origins; i.e., magnesium should also be released, to some extent, by SNe Ia and/or AGB stars, while oxygen is only enriched by CCSNe.

- The spatial and age trends cannot be easily disentangled, particularly for the thick disk objects, due to the paucity of available data. Our thin and thick disk samples, due to the difference in age of the component stars, can also be interpreted as two age groups with a boundary at $\sim 9 \mathrm{Gyr}$. The young group clearly follows negative radial gradients in $[\mathrm{O} / \mathrm{H}]$ and $[\mathrm{C} / \mathrm{O}]$ and positive vertical trends for $[\mathrm{O} / \mathrm{Fe}]$ and $[\mathrm{O} / \mathrm{Mg}]$. The second group shows almost flat radial trends and negative vertical gradients for $[\mathrm{O} / \mathrm{H}],[\mathrm{O} / \mathrm{Fe}]$, and $[\mathrm{O} / \mathrm{Mg}]$; the positive trends found for $[\mathrm{C} / \mathrm{O}]$, as mentioned in Section 4.3, should be taken with caution. Here $[\mathrm{O} / \mathrm{Fe}]$ and $[\mathrm{O} / \mathrm{Mg}]$ show positive trends with age for both the thin and thick disk stellar samples, with a smooth merger of the two trends for $[\mathrm{O} / \mathrm{Mg}]$, suggesting that this abundance ratio can be a good proxy of stellar ages. The $[\mathrm{O} / \mathrm{H}]$ trend of the thin disk stars is almost flat and suggests that the thin disk formed after most of the enrichment of oxygen in the interstellar medium by CCSNe had already occurred. The positive trends of $[\mathrm{O} / \mathrm{H}]$ and $[\mathrm{C} / \mathrm{O}]$ with age for the thick disk sample need further investigation (more objects) to clarify whether they are real or the result of a combination of statistical effects and the observed vertical gradient of $[\mathrm{O} / \mathrm{H}]$.

- The thin disk star trend of $[\mathrm{C} / \mathrm{O}]$ versus $[\mathrm{Fe} / \mathrm{H}]$ shows a negative value of $[\mathrm{C} / \mathrm{O}]$ at $[\mathrm{Fe} / \mathrm{H}] \simeq 0$, which implies that the Sun has a higher $\mathrm{C} / \mathrm{O}$ value than the solar neighborhood. We adopt a solar ratio of 0.54 (Grevesse et al. 2007), while our thin disk sample indicates a lower $\mathrm{C} / \mathrm{O}$ value of $\sim 0.45$. By using early-type B stars, Nieva \& Przybilla (2012) inferred $\mathrm{C} / \mathrm{O}=0.37$ for the mean present-day chemical composition of the cosmic matter in the solar neighborhood. They attributed this result, which is in qualitative agreement with ours, to an outward migration of the Sun in the galactic disk and the effects of Galactic chemical evolution and abundance gradients. The $\mathrm{C} / \mathrm{O}$ ratio, therefore, reveals imprints left from the Sun's original chemical composition.

We can conclude that our results confirm the hypothesis that CCSNe are the principal or, possibly, the only accountable source of enrichment of oxygen in the Galaxy disks and that $[\mathrm{O} / \mathrm{Mg}]$ can be a better indicator of stellar age than $[\mathrm{O} / \mathrm{Fe}]$.

The data presented in this paper, including the derived abundances and atmospheric parameter values, are part of the full data set from the GES. The spectra used here are a subsample of the over 200,000 reduced spectra of more than 100,000 stars observed and processed by the Gaia-ESO teams 
and are available through the ESO archive. The astrophysical parameters and abundances will be available shortly.

This work is based on data products from observations made with ESO telescopes at the La Silla Paranal Observatory under program ID 188.B-3002. These data products have been processed by the Cambridge Astronomy Survey Unit (CASU) at the Institute of Astronomy, University of Cambridge, and the FLAMES/UVES reduction team at INAF/Osservatorio Astrofisico di Arcetri. These data have been obtained from the GaiaESO Survey Data Archive, prepared and hosted by the Wide Field Astronomy Unit, Institute for Astronomy, University of Edinburgh, which is funded by the UK Science and Technology Facilities Council. This work was partly supported by the European Union FP7 program through ERC grant No. 320360 and the Leverhulme Trust through grant RPG-2012541. We acknowledge support from INAF and the Ministero dell' Istruzione, dell' Università e della Ricerca (MIUR), in the form of the grant "Premiale VLT 2012." The results presented here benefited from discussions held during the Gaia-ESO workshops and conferences supported by the European Science Foundation (ESF) through the GREAT Research Network Programme.

This work has made use of data from the European Space Agency (ESA) mission Gaia (https://www.cosmos.esa.int/ gaia), processed by the Gaia Data Processing and Analysis Consortium (DPAC; https://www.cosmos.esa.int/web/gaia/ dpac/consortium). Funding for the DPAC has been provided by national institutions, in particular the institutions participating in the Gaia Multilateral Agreement.

This work received partial financial support from the PRIN MIUR 2010-2011 project "The Chemical and dynamical Evolution of the Milky Way and Local Group Galaxies," prot. 2010LY5N2T, and the National Institute for Astrophysics (INAF) through grant PRIN-2014 ("The Gaia-ESO Survey").

M.C. is thankful for financial support from CONACyT grant CB-2015-256961. V.A. is supported by Fundação para a Ciência e Tecnologia (FCT) through national funds and by FEDER through COMPETE2020-Programa Operacional Competitividade e Internacionalização by these grants: UID/ FIS/04434/2019, UIDB/04434/2020, UIDP/04434/2020, PTDC/FIS-AST/32113/2017 and POCI-01-0145-FEDER032113, and PTDC/FIS-AST/28953/2017 and POCI-010145-FEDER-028953. V.A. also acknowledges support from the FCT through Investigador FCT contract No. IF/00650/ 2015/CP1273/CT0001. T.B. acknowledges financial support by grant No. 2018-04857 from the Swedish Research Council. U.H. acknowledges support from the Swedish National Space Agency (SNSA/Rymdstyrelsen).

This research used the facilities of the Italian Center for Astronomical Archive (IA2) operated by INAF.

Facilities: VLT:Kueyen, UVES.

Software: SPECTRUM (v2.76f; Gray \& Corbally 1994), ATLAS12 (Kurucz 2005).

\section{ORCID iDs}

Mariagrazia Franchini (i) https://orcid.org/0000-0001-5611-2333 Carlo Morossi iD https://orcid.org/0000-0002-3319-6375 Paolo Di Marcantonio (1) https://orcid.org/0000-0003-3168-2289 Miguel Chavez (BD https://orcid.org/0000-0003-0407-8115 Vardan Adibekyan (iD https://orcid.org/0000-0002-0601-6199 Thomas Bensby (1) https://orcid.org/0000-0003-3978-1409
Angela Bragaglia (D) https://orcid.org/0000-0002-0338-7883

Anais Gonneau (1) https://orcid.org/0000-0001-9091-5666

Ulrike Heiter (1) https://orcid.org/0000-0001-6825-1066

Georges Kordopatis (i) https://orcid.org/0000-0002-9035-3920

Laura Magrini (1) https://orcid.org/0000-0003-4486-6802

Donatella Romano (iD https://orcid.org/0000-0002-0845-6171

Luca Sbordone (i) https://orcid.org/0000-0002-2285-8708

Rodolfo Smiljanic (i) https://orcid.org/0000-0003-0942-7855

Gražina Tautvaišienė (iD https://orcid.org/0000-0001-

$7672-154 X$

Gerry Gilmore (iD https://orcid.org/0000-0003-4632-0213

Sofia Randich (1D) https://orcid.org/0000-0003-2438-0899

Amelia Bayo (iD https://orcid.org/0000-0001-7868-7031

Giovanni Carraro (i) https://orcid.org/0000-0002-0155-9434

Lorenzo Morbidelli (i) https://orcid.org/0000-0003-0304-9910

Simone Zaggia (1) https://orcid.org/0000-0001-6081-379X

\section{References}

Adibekyan, V. Z., Sousa, S. G., Santos, N. C., et al. 2012, A\&A, 545, A32 Akerman, C. J., Carigi, L., Nissen, P. E., Pettini, M., \& Asplund, M. 2004, A\&A, 414, 931

Allende Prieto, C., Beers, T. C., Wilhelm, R., et al. 2006, ApJ, 636, 804

Allende Prieto, C., Lambert, D. L., \& Asplund, M. 2001, ApJL, 556, L63

Amarsi, A. M., Asplund, M., Collet, R., \& Leenaarts, J. 2015, MNRAS, 454, L11

Amarsi, A. M., Asplund, M., Collet, R., \& Leenaarts, J. 2016, MNRAS, 455,3735

Amarsi, A. M., Nissen, P. E., \& Skúladóttir, Á. 2019, A\&A, 630, A104

Anders, F., Chiappini, C., Santiago, B. X., et al. 2014, A\&A, 564, A115

Asplund, M., Grevesse, N., Sauval, A. J., \& Scott, P. 2009, ARA\&A, 47, 481

Becker, S. A., \& Iben, I., Jr. 1979, ApJ, 232, 831

Bensby, T., \& Feltzing, S. 2006, MNRAS, 367, 1181

Bensby, T., Feltzing, S., \& Lundström, I. 2004, A\&A, 415, 155

Bensby, T., Feltzing, S., \& Oey, M. S. 2014, A\&A, 562, A71

Bertran de Lis, S., Allende Prieto, C., Majewski, S. R., et al. 2016, A\&A, 590, A74

Bertran de Lis, S., Delgado Mena, E., Adibekyan, V. Z., Santos, N. C., \& Sousa, S. G. 2015, A\&A, 576, A89

Boeche, C., Siebert, A., Piffl, T., et al. 2013, A\&A, 559, A59

Boeche, C., Siebert, A., Piffl, T., et al. 2014, A\&A, 568, A71

Boesgaard, A. M., King, J. R., Deliyannis, C. P., \& Vogt, S. S. 1999a, AJ, 117,492

Boesgaard, A. M., King, J. R., Deliyannis, C. P., \& Vogt, S. S. 1999b, AJ, 118,2542

Brewer, J. M., \& Fischer, D. A. 2016, ApJ, 831, 20

Brewer, J. M., Fischer, D. A., \& Madhusudhan, N. 2017, AJ, 153, 83

Buder, S., Lind, K., Ness, M. K., et al. 2019, A\&A, 624, A19

Burnside, R. G., Meriwether, J. W., \& Torr, M. R, J. 1977, P\&SS, 25, 985

Calura, F., \& Menci, N. 2009, MNRAS, 400, 1347

Carigi, L., Peimbert, M., Esteban, C., \& García-Rojas, J. 2005, ApJ, 623, 213

Castro, S., Gayoso, M., \& Rodríguez, C. 1997, JSSCh, 134, 227

Cescutti, G., Matteucci, F., François, P., \& Chiappini, C. 2007, A\&A, 462, 943

Cescutti, G., Matteucci, F., McWilliam, A., \& Chiappini, C. 2009, A\&A, 505,605

Chen, Y. Q., Zhao, G., Nissen, P. E., Bai, G. S., \& Qiu, H. M. 2003, ApJ, 591, 925

Cheng, J. Y., Rockosi, C. M., Morrison, H. L., et al. 2012, ApJ, 746, 149

Chiappini, C., Matteucci, F., \& Gratton, R. 1997, ApJ, 477, 765

Chiappini, C., Romano, D., \& Matteucci, F. 2003, MNRAS, 339, 63

De Silva, G. M., Freeman, K. C., Bland-Hawthorn, J., et al. 2015, MNRAS, 449, 2604

Delgado Mena, E., Israelian, G., González Hernández, J. I., et al. 2010, ApJ, 725,2349

Delgado Mena, E., Moya, A., Adibekyan, V., et al. 2019, A\&A, 624, A78 Ecuvillon, A., Israelian, G., Santos, N. C., et al. 2006, A\&A, 445, 633

Edvardsson, B., Andersen, J., Gustafsson, B., et al. 1993, A\&A, 500, 391

Esteban, C., García-Rojas, J., Peimbert, M., et al. 2005, ApJL, 618, L95

Franchini, M., Morossi, C., Di Marcantonio, P., et al. 2018, ApJ, 862, 146 Franchini, M., Morossi, C., Di Marcantonio, P., et al. 2020, ApJ, 888, 55 Gaia Collaboration, Katz, D., Antoja, T., et al. 2018, A\&A, 616, A11

Gaidos, E. 2015, ApJ, 804, 40 
Gilmore, G., Randich, S., Asplund, M., et al. 2012, Msngr, 147, 25 González Hernández, J. I., Bonifacio, P., Ludwig, H. G., et al. 2010, A\&A, 519, A46

Gray, R. O., \& Corbally, C. J. 1994, AJ, 107, 742

Grevesse, N., Asplund, M., \& Sauval, A. J. 2007, SSRv, 130, 105

Griffith, E., Johnson, J. A., \& Weinberg, D. H. 2019, ApJ, 886, 84

Grisoni, V., Spitoni, E., Matteucci, F., et al. 2017, MNRAS, 472, 3637

Gustafsson, B., Karlsson, T., Olsson, E., Edvardsson, B., \& Ryde, N. 1999, A\&A, 342, 426

Hayden, M. R., Bovy, J., Holtzman, J. A., et al. 2015, ApJ, 808, 132

Hayden, M. R., Recio-Blanco, A., de Laverny, P., et al. 2018, A\&A, 609, A79

Haywood, M., Di Matteo, P., Snaith, O., \& Lehnert, M. D. 2015, A\&A, 579, A5

Mikolaitis, Š., Hill, V., Recio-Blanco, A., et al. 2014, A\&A, 572, A33

Iben, I., Jr. 1964, ApJ, 140, 1631

Iben, I., Jr. 1965, ApJ, 142, 1447

Iben, I., Jr. 1967, ARA\&A, 5, 571

Israelian, G., García López, R. J., \& Rebolo, R. 1998, ApJ, 507, 805

Israelian, G., Rebolo, R., García López, R. J., et al. 2001a, ApJ, 551, 833

Israelian, G., Rebolo, R., García López, R. J., et al. 2001b, ApJ, 560, 535

Johansson, S., Litzén, U., Lundberg, H., \& Zhang, Z. 2003, ApJL, 584, L107

Jönsson, H., Allende Prieto, C., Holtzman, J. A., et al. 2018, AJ, 156, 126

Katz, D., Soubiran, C., Cayrel, R., et al. 2011, A\&A, 525, A90

Kordopatis, G., Wyse, R. F. G., Gilmore, G., et al. 2015, A\&A, 582, A122

Kurucz, R. L. 2005, MSAIS, 8, 14

Magrini, L., Randich, S., Kordopatis, G., et al. 2017, A\&A, 603, A2

Majewski, S. R., Schiavon, R. P., Frinchaboy, P. M., et al. 2017, AJ, 154, 94

Maoz, D., Mannucci, F., \& Brandt, T. D. 2012, MNRAS, 426, 3282

Matteucci, F., \& Chiappini, C. 2001, NewAR, 45, 567

Matteucci, F., \& Greggio, L. 1986, A\&A, 154, 279

Meléndez, J., Asplund, M., Gustafsson, B., \& Yong, D. 2009, ApJL, 704, L66

Meléndez, J., Barbuy, B., \& Spite, F. 2001, ApJ, 556, 858

Minchev, I., Chiappini, C., \& Martig, M. 2013, A\&A, 558, A9
Naiman, J. P., Pillepich, A., Springel, V., et al. 2018, MNRAS, 477, 1206

Nieva, M. F., \& Przybilla, N. 2012, A\&A, 539, A143

Nissen, P. E. 2013, A\&A, 552, A73

Nissen, P. E. 2015, A\&A, 579, A52

Nissen, P. E., Chen, Y. Q., Carigi, L., Schuster, W. J., \& Zhao, G. 2014, A\&A, 568, A25

Nissen, P. E., \& Edvardsson, B. 1992, A\&A, 261, 255

Nissen, P. E., \& Gustafsson, B. 2018, A\&ARv, 26, 6

Nissen, P. E., Primas, F., Asplund, M., \& Lambert, D. L. 2002, A\&A, 390, 235

Nomoto, K., Kobayashi, C., \& Tominaga, N. 2013, ARA\&A, 51, 457

Osterbrock, D. E., Fulbright, J. P., Martel, A. R., et al. 1996, PASP, 108, 277

Pavlenko, Y. V., Kaminsky, B. M., Jenkins, J. S., et al. 2019, A\&A, 621, A112

Petigura, E. A., \& Marcy, G. W. 2011, ApJ, 735, 41

Ramírez, I., Allende Prieto, C., \& Lambert, D. L. 2013, ApJ, 764, 78

Ramírez, I., Meléndez, J., \& Asplund, M. 2009, A\&A, 508, L17

Randich, S., Gilmore, G. \& Gaia-ESO Consortium 2013, Msngr, 154, 47

Recio-Blanco, A., de Laverny, P., Kordopatis, G., et al. 2014, A\&A, 567, A5

Reddy, B. E., Lambert, D. L., \& Allende Prieto, C. 2006, MNRAS, 367, 1329

Rocha-Pinto, H. J., Flynn, C., Scalo, J., et al. 2004, A\&A, 423, 517

Romano, D., Franchini, M., Grisoni, V., et al. 2020, A\&A, 639, A37

Romano, D., Karakas, A. I., Tosi, M., \& Matteucci, F. 2010, A\&A, 522, A32

Rudolph, A. L., Fich, M., Bell, G. R., et al. 2006, ApJS, 162, 346

Schlesinger, K. J., Johnson, J. A., Rockosi, C. M., et al. 2014, ApJ, 791, 112

Snaith, O., Haywood, M., Di Matteo, P., et al. 2015, A\&A, 578, A87

Spitoni, E., Silva Aguirre, V., Matteucci, F., Calura, F., \& Grisoni, V. 2019, A\&A, 623, A60

Stonkutè, E., Chorniy, Y., Tautvaišienè, G., et al. 2020, AJ, 159, 90

Ventura, P., D'Antona, F., Imbriani, G., et al. 2018, MNRAS, 477, 438

Wang, L.-L. 2019, PASP, 131, 077001

Weinberg, D. H., Holtzman, J. A., Hasselquist, S., et al. 2019, ApJ, 874, 102

Wenger, T. V., Balser, D. S., Anderson, L. D., \& Bania, T. M. 2019, ApJ, 887,114

Woosley, S. E., \& Weaver, T. A. 1995, ApJS, 101, 181 